\title{
PATTERN SIZE IN GAUSSIAN FIELDS FROM SPINODAL DECOMPOSITION*
}

\author{
LUIGI AMEDEO BIANCHI ${ }^{\dagger}$, DIRK BLÖMKER ${ }^{\ddagger}$, AND PHILIPP WACKER W $^{\S}$
}

\begin{abstract}
We study the two-dimensional snake-like pattern that arises at the onset of phase separation of alloys described by spinodal decomposition in the Cahn-Hilliard model. These are somewhat universal patterns due to an overlay of the most unstable pattern, which are eigenfunctions of the Laplacian all with a similar wave-number. Similar structures appear in other models like reaction-diffusion systems describing animal coats' patterns, hill formation in surface growth, or vegetation patterns in desertification. In order to study the early stages of spinodal decomposition we focus on the linearized equation. Our main result studies random functions given by cosine Fourier series with independent Gaussian coefficients, which dominate the dynamics in the Cahn-Hilliard model. This is not a cosine process, as the sum is taken over domains in Fourier space that not only grow and scale with a parameter of order $1 / \varepsilon$ for a small $0<\varepsilon \ll 1$, but also move to infinity for $\varepsilon \rightarrow 0$. Moreover, the model under consideration is as a random field at a fixed time neither stationary nor isotropic. To study the pattern size of nodal domains we consider the density of zeros on any straight line through the spatial domain. Using a theorem by Edelman and Kostlan and weighted ergodic theorems that ensure the convergence of the moving sums, we show that the average distance of zeros is asymptotically of order $\varepsilon$ with a precisely given constant.
\end{abstract}

Key words. Cahn-Hillard equation, pattern size, Gaussian fields, ergodic theorem

AMS subject classifications. 60H15, 60H05, 60G15, 60G60

DOI. $10.1137 / 15 \mathrm{M} 1052081$

1. Introduction. We are interested in studying the patterns that form in the solution of the stochastic Cahn-Hilliard equation during the separation process called spinodal decomposition. This equation was originally introduced in its deterministic version in [10] and [11] and later in its stochastic version by [12]. It models the relative concentration of two components in an alloy after quenching an initially homogeneous mixture and exhibits a spontaneous phase separation. Here we are focusing on the onset of this separation process. The dynamics of this separation was already studied for the deterministic case with possibly random initial conditions in $[28,29,35]$ and for the stochastic case in $[6,7,8]$. In all these publications it was shown that the onset of spinodal decomposition for a surprisingly long time is well described by the linearized model. Nevertheless all these publications could only address the pattern size appearing in the phase separation as an overlay of functions with a similar wavenumber.

*Received by the editors December 9, 2015; accepted for publication (in revised form) February 24, 2017; published electronically August 17, 2017.

http://www.siam.org/journals/siap/77-4/M105208.html

Funding: The first author's work was supported by the German Science Foundation (DFG), grant BL 535/9-2, "Mehrskalenanalyse stochastischer partieller Differentialgleichungen (SPDEs)." The third author's work was supported by a Cusanuswerk scholarship.

$\dagger$ Institut für Mathematik, Technische Universität Berlin, Berlin, Germany. Previous address: Institut für Mathematik, Universität Augsburg, Augsburg, Germany (bianchi@math.tu-berlin.de, http://page.math.tu-berlin.de/ bianchi/).

${ }^{\ddagger}$ Institut für Mathematik, Universität Augsburg, Augsburg, Germany (dirk.bloemker@math.uniaugsburg.de, http://www.math.uni-augsburg.de/prof/ana/arbeitsgruppe/bloemker/).

$\S$ Department Mathematik, Friedrich-Alexander-Universität Erlangen, Erlangen, Germany (wacker@math.fau.de, https://www.mso.math.fau.de/applied-mathematics-1/staff-members/ wacker-philipp/). 
Similar structures and patterns appear in many other situations, for both stochastic and deterministic models. One example are the patterns in coats of animals like zebras or tigers [31], where the underlying system is a reaction-diffusion system with a Turing instability, as argued by Sander and Wanner in [36]. Another occurrence is the vegetation patterns in desertification like the one observed for the tiger bush in Africa, as studied, for example, by Siero and others in [37, 38]. In those papers they provide a mathematical study of the emergence of pattern on an unbounded domain in the extended Klausmeier model, which is similar to a reaction-diffusion system.

More similar to the Cahn-Hilliard setting is the stochastic Swift-Hohenberg equation (see, for example, the review [14]). A result very much related to spinodal decomposition is given by Blömker and Maier-Paape in [5]. The main difference is that the instability is very weak and significantly fewer Fourier modes determine the pattern.

Another example is a stochastic partial differential equation arising in the theory of surface growth [32, 25], which is also used in ion sputtering [30]. Here hills form on an initially flat surface and in the early stages the model exhibits patterns similar to spinodal decomposition. For details see the review article [9].

In all these examples, as studied in the Cahn-Hilliard equation both stochastic and deterministic, the characteristic snake-like pattern that appears initially is mainly due to composition of the most unstable pattern that dominates the linearized dynamics. These are eigenfunctions of the Laplacian all with a similar wavelength. In the early stages of the pattern formation process the nonlinearity does not yet play a significant role, and the solution is described well by a high dimensional space of the most unstable pattern.

In order to outline this idea, we focus in the following sections on the stochastic Cahn-Hilliard equation on the square. Moreover, for simplicity we focus on the stochastic model, where the most unstable patterns are randomly weighted. In the case of patterns at a fixed time, which we study here, similar results are true if we take the deterministic model with Gaussian random initial conditions.

The main question that we want to answer rigorously in this paper is the following: At a fixed time early in the separation process, what is the characteristic thickness of the pattern (i.e., the snake-like structures)?

To address this, we consider the pattern at a fixed time and draw an arbitrary straight line across the domain. We then count the number of zeros of the pattern along this line to determine the pattern size. Using a result given by Edelman and Kostlan in [19] we can also obtain rigorous results for the expected number of zeros along one line, which we can then evaluate explicitly in the asymptotic limit of small interaction length.

In the context of spinodal decomposition, for the deterministic model a partial result for the pattern size was obtained by Maier-Paape and Wanner in [28], where they tried to estimate the size of balls that would fit into the nodal domain of a pattern. A numerical computation of Betti numbers of nodal domains to get topological informatuon about the pattern was performed in a series of papers $[20,16,17]$.

Moreover the spatial structure of nodal domains for Gaussian random fields is the topic of numerous recent publications, which we do not try to survey in full detail. Under the assumption of spatial stationarity and isotropy, which we do not have in our model, there are asymptotic results on the numer of zeros along lines. See, for example, [18, 27]. Minkowski functionals are also treated; see [2] for a review, or [39] for references to asymptotic results of the number of connected components. We comment on these approaches later on in more detail. 
1.1. The Cahn-Hilliard-Cook equation. The stochastic Cahn-Hilliard (or Cahn-Hilliard-Cook) equation was introduced by Cook in [12] as a stochastic modification of the originally deterministic model. It can be written as follows:

$$
\partial_{t} u=-\Delta\left(\varepsilon^{2} \Delta u+\mathcal{F}(u)\right)+\partial_{t} W
$$

where the noise $\partial_{t} W$ is the derivative of a $Q$-Wiener process and $\mathcal{F}$ is the derivative of a double well potential, where a standard choice is $\mathcal{F}(u)=u-u^{3}$, although the true nonlinearity introduced by Cahn and Hilliard should exhibit logarithmic poles. We consider it on the square domain $[0,1]^{2}$, with Neumann boundary conditions $\partial_{\nu} u=\partial_{\nu} \Delta u$. In the physical model of alloys, $u$ models the rescaled concentration of one component, and the extreme values $u= \pm 1$ correspond to $0 \%$ and $100 \%$ concentration of the first component in any point.

The canonical initial condition for the phase separation in spinodal decomposition is a homogeneous concentration

$$
u(0, \cdot) \approx m,
$$

which is constant on the whole domain.

Due to the presence of the noise in the equation, which is studied here, or to the presence of random fluctuations in the initial conditions, almost immediately the homogeneous picture is broken and decomposition starts playing its role. The snakelike pattern appears and persists, giving place to situations like the one simulated in Figure 1. Our aim now is to describe the pattern at a fixed time in the early onset of separation.

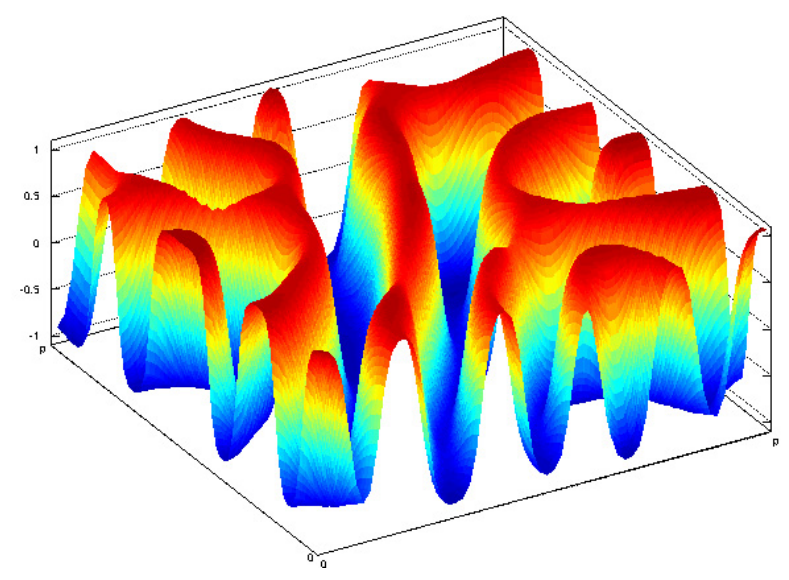

FIG. 1. Pattern in spinodal decomposition. The snake-like pattern appears as the nodal domains in the level set of the average concentration, which is here $m=0$.

For the Cahn-Hilliard equation, one can consider the set of the most unstable eigenvalues, which dominate the dynamics for a long time, also called the "strongly unstable subspace." This was studied in the deterministic model by Maier-Paape and Wanner in $[28,29]$ and later in the stochastic setting by Blömker, Maier-Paape, and Wanner in $[8,6]$ (see also the review [7]). All results relied on the approximation of the nonlinear dynamics via linearization in the early stages of the separation process. Later Sander and Wanner extended in [35] this approximation result by linearization to unexpectedly large radii, at least for the deterministic model.

In [20] Betti-numbers of nodal domains were numerically computed, and after the initial formation of the pattern the deterministic model with random initial conditions 
exhibits a short, quite unnatural increase of pattern complexity, which is not present in the stochastic model. This is a quite interesting observation that the evolution of pattern complexity is different in the deterministic model with random initial conditions or the stochastic model with constant initial condition, but we will focus on the onset of pattern formation before this stage.

1.2. Linearized Cahn-Hilliard-Cook equation. While the Cahn-Hilliard equation is highly nonlinear, its dynamics at the onset of pattern formation is reasonably well approximated by the linearized equation, which even holds true for quite long times up to unexpectedly large solutions, as shown in [35, 40]. So let us discuss the linearized system (around the spatially constant initial conditions $u(0, \cdot)=m$ ) for the deviation $v=u-m$. This will motivate why we study random cosine series later.

Such linearized system can be written as

$$
\partial_{t} v=A v+\partial_{t} W, \quad v(0)=0,
$$

where $A=-\varepsilon^{2} \Delta^{2}-\Delta \mathcal{F}^{\prime}(m)$ is a self-adjoint linear operator having a complete orthonormal system of eigenfunctions.

In our study we focus on the simple domain $[0,1]^{2}$ to avoid additional pattern complexity introduced by a complicated shape of the boundary. The $L^{2}$-basis on the square is made of cosine functions

$$
e_{k, l}(x)=C \cos (k \pi x) \cos (l \pi y)
$$

with $A e_{k, l}=\lambda_{k, l} e_{k, l}$, where

$$
\lambda_{k, l}=-\varepsilon^{2}\left(k^{2}+l^{2}\right)^{2} \pi^{4}+\left(k^{2}+l^{2}\right) \pi^{2} \mathcal{F}^{\prime}(m) .
$$

The solution to (1) is given by the stochastic convolution (see [15]), which simplifies here to a cosine series with random coefficients,

$$
W_{A}(t)=\sum_{k, l \in \mathbb{N}} \int_{0}^{t} e^{(t-s) A} \mathrm{~d} W(s)=\sum_{k, l \in \mathbb{N}} \alpha_{k, l} \int_{0}^{t} e^{(t-s) \lambda_{k, l}} \mathrm{~d} B_{k, l}(s) e_{k, l},
$$

where the $B_{k}$ are independent Brownian motions and the $Q$-Wiener process $W$ has a joint eigenbasis with $A$ such that $Q e_{k, l}=\alpha_{k, l}^{2} e_{k, l}$. This is an usual but quite strong assumption that $Q$ commutes with $A$. As this is only our motivating example, for simplicity of presentation we do not enter this discussion here. Some details and further references can be found in [7]. One also could think of space-time white noise, where $Q$ is the identity and thus all $\alpha_{k, l}$ are one.

In the case where $m$ is in the spinodal region defined by $\mathcal{F}^{\prime}(m)>0$ a simple calculation shows that the upper bound of the eigenvalues $\lambda_{k, l}$ is given by

$$
\lambda_{\max }=\frac{1}{4} \varepsilon^{-2}\left(\mathcal{F}^{\prime}(m)\right)^{2} .
$$

The results on the early stages of spinodal decomposition (see, for example, [6]) show that the eigenfunctions of the most unstable eigenvalues form a strongly unstable subspace also called "strong subspace", for short, that dominates the dynamics of the Cahn-Hilliard equation.

To be more precise this space is defined for any fixed $\gamma \in(0,1)$ close to 1 by taking all eigenfunctions $e_{k, l}$ with eigenvalues satisfying $\lambda_{k, l}>\gamma \lambda_{\max }$. Thus in Fourier space by the wave-vectors

$$
R_{\varepsilon}^{\gamma}:=\left\{(k, l) \in \mathbb{N}^{2}: \lambda_{k, l}>\gamma \lambda_{\max }\right\} .
$$


In particular, in the two-dimensional setting we consider, the set $R_{\varepsilon}^{\gamma}$ is a quarter-ring in the $\mathbb{N}^{2}$ lattice, which is easily seen by discussing the inequality $-\varepsilon^{2} z^{2} \pi^{4}+z \pi^{2} \mathcal{F}^{\prime}(m)>$ $\gamma \lambda_{\max }$. This is nonempty only if $\varepsilon$ is not too large, and moreover it contains $\mathcal{O}\left(\varepsilon^{-2}\right)$ many wave-vectors for $\varepsilon \rightarrow 0$. In the following we do not always specify the explicit dependence of $R_{\varepsilon}^{\gamma}$ on $\gamma$ and write $R_{\varepsilon}$ for short. Note that this set not only is growing in size for $\varepsilon \rightarrow 0$ but also moves as a whole toward infinity.

As already outlined, as long as the solution is not too large, the dynamics of the nonlinear Cahn-Hilliard equation is dominated by the projection $P_{R_{\varepsilon}}$ of the stochastic convolution on the strong subspace. By this we mean the restriction of the Fourier series to wave-vectors in $R_{\varepsilon}$, which is given by

$$
P_{R_{\varepsilon}} W_{A}(t)=\sum_{(k, l) \in R_{\varepsilon}} \alpha_{k, l} c_{k, l} e_{k, l} \quad \text { with } \quad c_{k, l}=\int_{0}^{t} e^{(t-s) \lambda_{k, l}} \mathrm{~d} B_{k, l}(s) .
$$

The random variables in the family $\left\{c_{k, l}\right\}_{(k, l) \in R_{\varepsilon}}$ are by definition independent centered Gaussians. By Itō-isometry the variance of $c_{k, l}$ is

$$
\mathbb{E} c_{k, l}^{2}=\int_{0}^{t} e^{(t-s) 2 \lambda_{k, l}} \mathrm{~d} s=\frac{1}{2 \lambda_{k, l}}\left(1-e^{-2 \lambda_{k, l} t}\right) \approx \frac{1}{2 \lambda_{k, l}}=\mathcal{O}\left(\varepsilon^{2}\right)
$$

for times $t \approx \varepsilon^{2}$, which is close to the time-scale on which the first phase of spinodal decomposition was described (see [6]). We also used that in the strongly unstable subspace $\lambda_{k, l} \in(1-\gamma, 1] \lambda_{\max }$ and thus $\lambda_{k, l}$ is of the order $\varepsilon^{-2}$.

Hence after linearization and projection via $P_{R_{\varepsilon}}$, the solution at a fixed time $t$ seems to be well approximated by

$$
u(t, x, y) \approx m+v(t, x, y) \approx m+\sum_{(k, l) \in R_{\varepsilon}} c_{k, l} \cdot \cos (k \pi x) \cos (l \pi y),
$$

where the $c_{k, l}$ are independent centered Gaussians with similar variances. This motivates the choice of our toy model for studying the pattern at a fixed time $t$ early in the onset of spinodal decomposition. We will present the model in more detail in the next subsection. There we make an additional simplification by fixing for ease of presentation all the variances of the coefficients to be the same, although they are only of the same order in $\varepsilon$.

1.3. Random Fourier series. For the main part of the paper we consider the random function

$$
f(x, y)=\sum_{(k, l) \in R_{\varepsilon}} c_{k, l} \cdot \cos (k \pi x) \cos (l \pi y)
$$

on the unit square $x, y \in[0,1]^{2}$, with the random coefficients $c_{k, l}$ being independent and identically distributed centered Gaussian random variables. Later we discuss also the impact of different domains $R_{\varepsilon}$ in Fourier space, and not only the ring discussed below.

As we discussed in the previous subsection, this is a simplified model for the stochastic Cahn-Hilliard equation at a fixed time early in the onset of pattern formation. First it simplifies by using the approximation (2) of the stochastic Cahn-Hilliard equation, and second by assuming that all coefficients are identically distributed, although they are only of the same order in $\varepsilon$. We furthermore set the variance of the 
random coefficients to be 1 , i.e., $c_{k, l} \sim N(0,1)$. The actual size of the function is not important when we study the nodal domain.

As our main example we consider the Cahn-Hilliard equation and fix the average mass to be $m=0$. If we consider different $m$ we would only change the shape of $R_{\varepsilon}$ and the value of various constants. In the case $m=0$ the subset $R_{\varepsilon}$ of strongly unstable modes is given by

$$
R_{\varepsilon}=\left\{(k, l) \in \mathbb{N}^{2} \mid \alpha_{\ominus}<\sqrt{(k \varepsilon)^{2}+(l \varepsilon)^{2}}<\alpha_{\oplus}\right\},
$$

where from $\lambda_{k, l}>\gamma \lambda_{\max }$ with $m=0$ we obtain the parameters

$$
\alpha_{\oplus}=\sqrt{\frac{1+\sqrt{1-\gamma}}{2 \pi^{2}}} \text { and } \quad \alpha_{\ominus}=\sqrt{\frac{1-\sqrt{1-\gamma}}{2 \pi^{2}}} \text { with } \quad \gamma \in(0,1) .
$$

Note that with $z=k^{2}+l^{2}$ for $m=0$ we have $\lambda_{k, l}=-\varepsilon^{2} z^{2} \pi^{4}+z \pi^{2}$ and thus $\lambda_{\max }=1 / 4 \varepsilon^{2}$.

Although the model (3) is somewhat reminiscent of the cosine process (see, e.g., [1]) or the random wave model (see, e.g., [18]), where questions related to the structure of nodal domains were discussed, it is fundamentally different in the sense that it is neither spatially stationary nor isotropic, which would imply that the law of $f$ is invariant under translation or rotation. But due to the Neumann boundary conditions on a square, the law of the random function $f$ (as a function extended to $\mathbb{R}^{2}$ ) changes under rotation around angles not being a multiple of 90 degrees. Moreover, as the normal derivative is always zero at the boundary of the square, the law of $f$ is also not invariant under arbitrary translations.

Let us finally remark that it is an easy calculation to show that $f(x)$ is a centered Gaussian with covariance

$$
\mathbb{E} f(x) f(y)=q(x+y)+q(x-y) \quad \text { for } \quad q(z)=\frac{1}{2} \sum_{(k, l) \in R_{\varepsilon}} \cos (k \pi z) \cos (l \pi z)
$$

which is also not invariant under arbitrary translation and rotation of the $(x, y)$-plane.

1.4. Pattern size. A simulation of (3) with $m=0$ and $\gamma=0.8$ is presented in Figure 2. We can see the snake-like patterns which are characteristic of the CahnHilliard model. It appears from the numerical simulations that the thickness of the

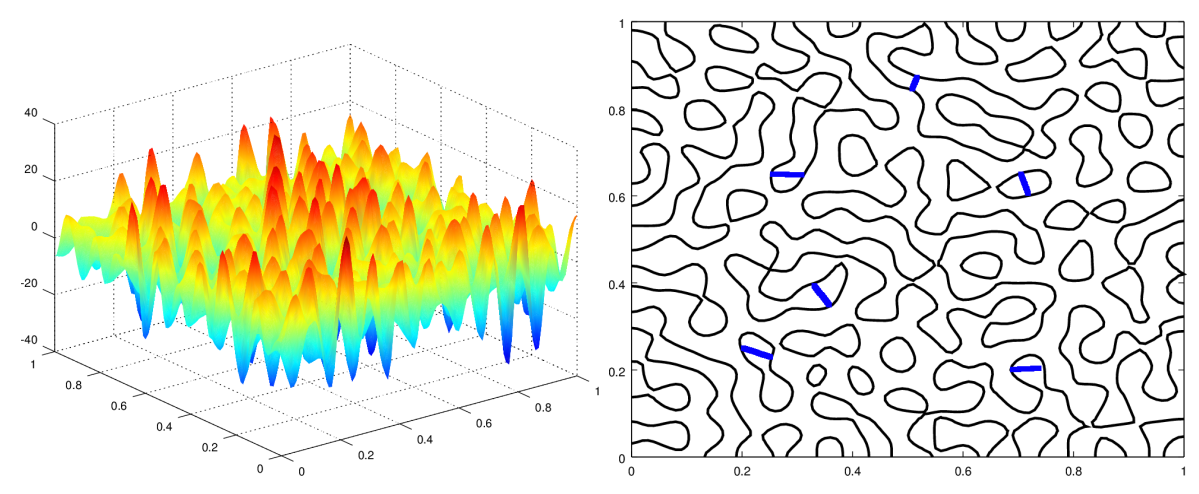

FIG. 2. A simulation of the function $f$ for $\gamma=0.8$ with a plot of its zero-level set. On the latter we picked some measurements of the pattern thickness. Empirically, the average distance is $2 \pi \varepsilon$. There is of course some variation and there are areas and directions where the thickness is much higher or lower. 


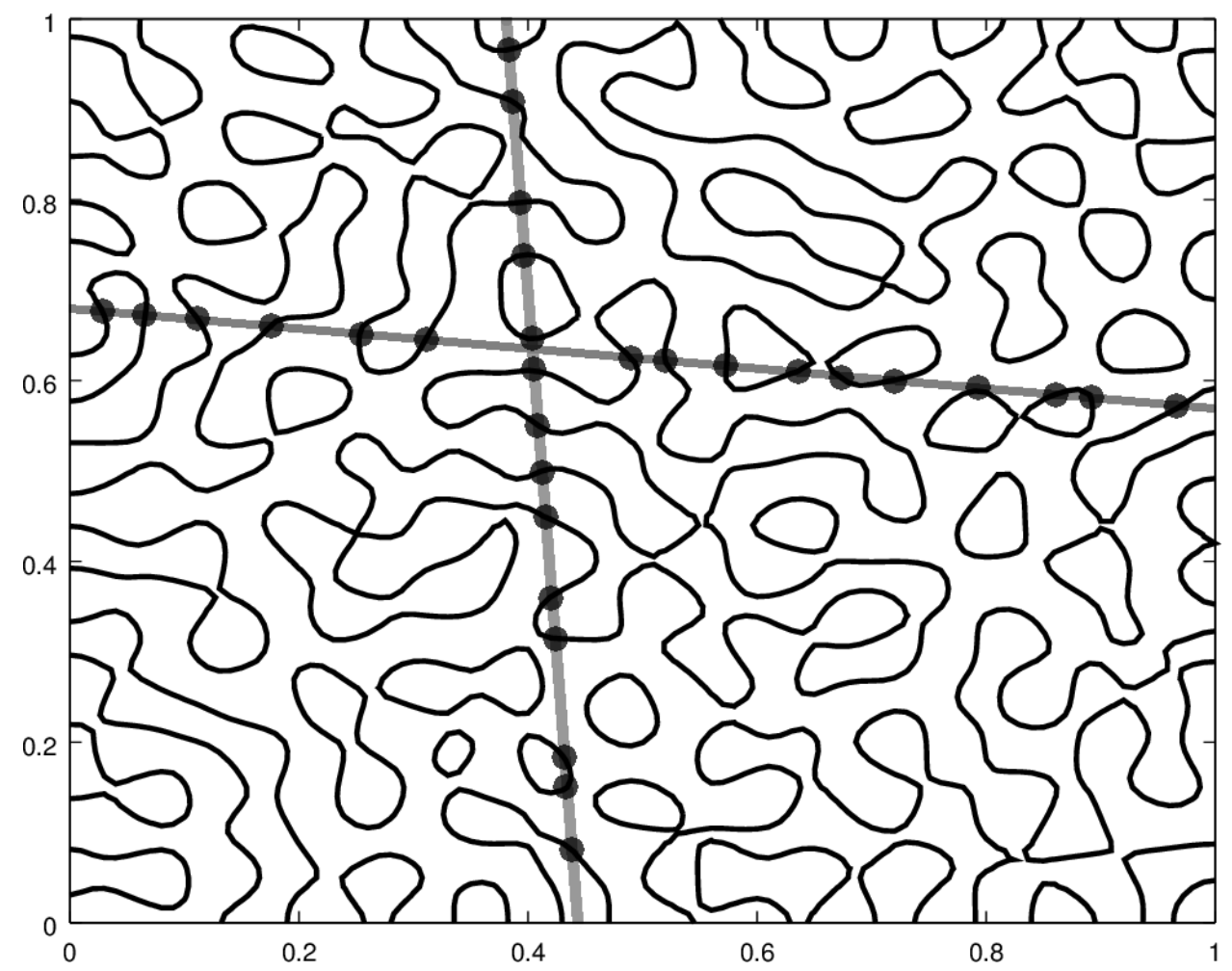

FIG. 3. Pattern size computation of the function $f$ by inserting arbitrary lines through the spatial domain $[0,1]^{2}$, where we represent the zero level sets for our model in the Fourier domain $R_{0.01}^{0.8}$. The zeros are order $\varepsilon$ apart, on average.

structures is somewhat proportional to $\varepsilon$, as shown in Figure 2. Here we propose to measure such thickness as the distance between consecutive zeros of the solution considered on an arbitrary line through the domain, as exhibited in Figure 3.

This definition of pattern size intuitively seems to be the right one. On the other hand the following fact is frequently stated in the literature: as by definition the dominating Fourier space $R_{\varepsilon}$ consists of wave-vectors $(k, l) \in R_{\varepsilon}$ that are all of the order $\varepsilon^{-1}$ in norm for small $\varepsilon$, the overall pattern size is of the order $\varepsilon$.

Although this line of reasoning sounds very plausible, and it is easily proved for a one-dimensional problem, in two dimensions the wave-vector consists of two frequencies that can vary vastly, and it is not clear why the average number of zeros along a line should scale with $\varepsilon$.

Moreover we will also see later in the main results that, when counting zeros on arbitrary straight lines, only the scaling of the upper bound for the wave-number seems to be essential. Let us remark that for spatially stationary and isotropic Gaussian fields there are quite a few publications on the average density of zeros along lines. They date back to the work of Rice [33] or Longuet-Higgins [27], [26]. See also Dennis [18]. Their approach yields qualitatively analogous results of a pattern size of order $\varepsilon$ for models given by cosine series similar to (3), but none of those methods seem to apply straightforwardly to (3), in that they usually assume spatial isotropy and spatial stationarity. 
A rigorous quantification of the geometry and topology of Cahn-Hilliard-Cook patterns is still an open problem, though. The first attempt in the setting of spinodal decomposition by Maier-Paape and Wanner in [28] yielded a partial result by bounding the radii of balls that would fit into the nodal domain. That strategy worked completely only in the setting of [5], where the ring $R_{\varepsilon}$ was still growing, but its size was smaller by a small power in $\varepsilon$.

As already mentioned in the previous subsection, recent numerical work on the number of components in the pattern has been done, using rigorous methods from computational algebraic topology to compute the Betti numbers, by Gameiro, Mischaikow, and Wanner in [20] or similarly by Day, et al. in [16, 17]. See also Guo and Hwang [23] and Sander and Tatum [34] for additional results on the pattern in Cahn-Hilliard equation. Let us recall that Betti numbers for the two-dimensional nodal domain count the number of connected components and the number of holes.

For general stationary or isotropic Gaussian fields there is also some work on Minkowski functionals measuring the area and the number of connected components of the nodal domain as well as the length of the set of level 0. See [2] for a collection of results. But even the precise constant in the asymptotic behavior of the number of connected components seems to be still an open problem. See [39] for further references. None of these results seems to fit to (3) anyway, not just because of the assumptions on isotropy and stationarity, but also because we are summing over index sets that not only are growing but also moving, and thus the asymptotic limit is not clear.

2. Main result and structure of the paper. The question we aim to rigorously answer is as follows.

QUESTION 2.1. What is the characteristic thickness of the pattern (i.e., snake-like structures) in our model $f(x, y)$ defined in (3), on the unit square $(x, y) \in[0,1]^{2}$ ?

In Figure 2 we can see that the average thickness appears to be $2 \pi \varepsilon$, which we will prove to be the case.

To address Question 2.1, we take the following approach: we draw a straight line across the unit square and we count the (average) number of zeros of $f$ on that segment; see Figure 3. Let us remark that if a nodal line coincides for some interval with the straight line, then we count this as infinity. We will see later in the main result that this is an event with probability zero, as the expected number of zeros will always be finite.

We divide the length of the segment by the number of zeros, obtaining the average distance between zeros, that is, the average pattern size. So the problem reduces to counting the number of zeros of $f$, a random function, and a way of doing this is provided (in a more general form than reported here) by Edelman and Kostlan in [19].

ThEOREM 2.2 (number of real zeros of a random function). Let

$$
v(x)=\left(f_{0}(x), \ldots, f_{n}(x)\right)^{T}
$$

be any collection of differentiable functions and $c_{0}, \ldots, c_{n}$ be independent and identically distributed Gaussians centered in 0. Given the function

$$
h(x)=\sum_{k=0}^{n} c_{k} \cdot f_{k}(x)
$$


the expected number of real zeros of $h$ on $I$ is

$$
\int_{I} \delta(x) \mathrm{d} x
$$

where

$$
\delta(x)=\frac{1}{\pi}\left\|\frac{\mathrm{d}}{\mathrm{d} x} w(x)\right\|_{\mathbb{R}^{n}} \quad \text { and } \quad w(x)=\frac{v(x)}{\|v(x)\|_{\mathbb{R}^{n}}} .
$$

Due to the fact that the average number is given by an integral over $\delta$, we call $\delta$ the density of zeros of $h$.

The proof of this theorem is given in [19]. The key idea of the much longer proof is that $x$ is a zero of $f$ if the vectors $\left(c_{k}\right)_{k}$ and $\left(f_{k}(x)\right)_{k}$ are orthogonal. Thus after normalization all admissible $\left(c_{k}\right)_{k}$ form an equator on the unit sphere, and one can translate the problem of counting zeros to determining the area covered by these equators (counted with multiplicities). Then one uses that the area is proportional to the derivative of the curve $x \mapsto\left(f_{k}(x)\right)_{k}$. Finally note that the argument for turning Gaussian probabilities into the calculation of area is the fact that a standard Gaussian vector after normalization induces a uniform distribution on the sphere.

Theorem 2.2 holds for functions $h$ on the real line, so we need to translate our two-dimensional problem $f$ to an equivalent one formulated on a line. In the following for simplicity first we consider $f$ constrained on an arbitrary horizontal line at height $\tau$, which is

$$
L_{\tau}=\{(x, \tau): x \in[0,1]\} \quad \text { for } \quad \tau \in[0,1] .
$$

We obtain a function of one variable and can apply Theorem 2.2. The generalization to arbitrary (nonhorizontal) lines as the ones depicted in Figure 3 is a straightforward generalization and is discussed in section 6 but the underlying idea does not change.

We need to introduce, in the spirit of Theorem 2.2, the following notation:

$$
w_{\tau}(x)=\left(\frac{\cos (k \pi x) \cos (l \pi \tau)}{\sqrt{\sum_{(m, n) \in R_{\varepsilon}} \cos ^{2}(m \pi x) \cos ^{2}(n \pi \tau)}}\right)_{(k, l) \in R_{\varepsilon}} .
$$

Note that although we parametrize $w_{\tau}(x)$ over the two-dimensional set $R_{\varepsilon}$, we consider it as a vector in $\mathbb{R}^{N_{\varepsilon}}$, where $N_{\varepsilon}$ is the size of $R_{\varepsilon}$. Moreover, we define

$$
W_{\tau}(x)=\left\|\left(\frac{\mathrm{d}}{\mathrm{d} x} w_{\tau}(x)\right)_{(k, l) \in R_{\varepsilon}}\right\|^{2}=\frac{S_{3}}{S_{1}}-\left(\frac{S_{2}}{S_{1}}\right)^{2},
$$

where we have

$$
\begin{aligned}
& S_{1}=\sum_{m, n \in R_{\varepsilon}} \cos ^{2}(m \pi x) \cos ^{2}(n \pi \tau), \\
& S_{2}=\sum_{m, n \in R_{\varepsilon}} m \pi \cos (m \pi x) \sin (m \pi x) \cos ^{2}(n \pi \tau), \\
& S_{3}=\sum_{m, n \in R_{\varepsilon}} m^{2} \pi^{2} \sin ^{2}(m \pi x) \cos ^{2}(n \pi \tau) .
\end{aligned}
$$

The main result for horizontal lines in the case of a ring $R_{\varepsilon}$ in Fourier space is the following one, which is stated in terms of $W_{\tau}(x)=\pi^{2} \delta(x)^{2}$ and gives the asymptotic behavior for small $\varepsilon$ of the densities of zeros. 

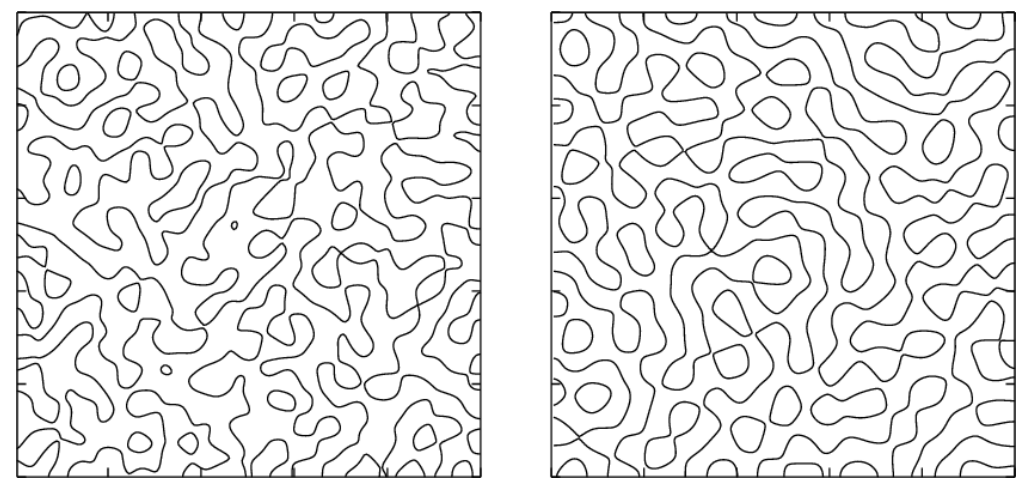

FIG. 4. Patterns generated in the Fourier domains $R_{0.01}^{0.1}$ (left) and $R_{0.01}^{0.9}$ (right).

Theorem 2.3. For any $\gamma \in(0,1)$ and any horizontal line $L_{\tau}$ for $x, \tau \in(0,1)$ the function $W_{\tau}(x)$ defined on $L_{\tau}$ behaves asymptotically as $(2 \varepsilon)^{-2}$ for $\varepsilon \rightarrow 0$.

This means in particular that the density of zeros is $\delta(x)=(2 \pi \varepsilon)^{-1}$.

Thus the mean pattern size (i.e., the average distance between zeros on any horizontal line of length 1 ) is given by $2 \pi \varepsilon$.

Remark 2.4. It is a remarkable fact that the result of Theorem 2.3 is independent of $\gamma$, because the number of Fourier modes involved is much smaller for $\gamma \approx 1$ than for $\gamma \approx 0$. As we can see in Figure 4, while the average asymptotic pattern size along lines remains the same, the domain with higher $\gamma$ looks more organized. The pattern seems to be "more regular" in some sense that our method cannot detect.

We will prove Theorem 2.3 later in section 3, where Lemma 3.7 shows

$$
\lim _{\varepsilon \rightarrow 0} \varepsilon \frac{S_{2}}{S_{1}}=0, \quad \quad \lim _{\varepsilon \rightarrow 0} \varepsilon^{2} \frac{S_{3}}{S_{1}}=\frac{1}{4} .
$$

The main idea behind the proof is that the sums $S_{i}, i=1,2,3$, can be calculated in the limit via ergodic-type theorems.

Remark 2.5. We always fix $x, \tau \in(0,1)$ for the horizontal lines, as the functions behave a little differently on the borders. If we have $\tau=0,1$, we lose all the terms in $\tau$, which become identically 1 , but we obtain anyway the same result. The cases $x=0,1$ are not interesting, as the sin-function is always zero and thus $W_{\tau}(0)=W_{\tau}(1)=0$. But since we are looking for a density in terms of $x$, considering it in a single point does not provide any useful information.

After the proof of Theorem 2.3 for the ring in Fourier space and the horizontal line we present several extensions of the result. In section 4 we state the generalization of the main result to general domains in Fourier space different from the quarter-ring and give a few explicit examples that the main result is still true, although the patterns appearing might look quite different.

As our result is a purely asymptotic one, we give a few examples in section 4, where the number of zeros agrees very well with the asymptotic prediction even for moderate $\varepsilon$. In section 5 , we calculate numerically the functions $\delta(x)$ or $W_{\tau}(x)$ for different values of $\varepsilon$. There (cf. also Figure 8) we see already for moderate $\varepsilon$ a fast and uniform convergence apart from any small layer at the boundary.

Finally in section 6 we briefly discuss the case of sloped lines, which is a straightforward generalization, up to some additional technicalities. In the appendix we show 
that we can establish convergence in the rational case not treated in the proof, where we cannot use Birkhoff's ergodic theorem.

Let us comment on further generalizations that are within the scope of this approach. First we can easily incorporate the case where the Gaussians are not all identically distributed. For instance, when the noise is diagonal w.r.t. the cosine modes, we just obtain additional weights in the main result, coming from the eigenvalues of the covariance operator of the noise.

For different domains in Fourier space we always get the same order of zeros on any line through the domain, but as we will see in Figure 7 the pattern might look quite different. Further characterizations using Betti numbers as in [20], the Minkowski functionals as in [2], or bounds on the radii of balls in the nodal domains as in [28] might help us to understand why the pattern in spinodal decomposition is so special, as it appears to be quite regular, but not too symmetric.

Moreover, we believe that the result on the square $[0,1]^{2}$ is not that special, and with the same method we should be able to treat three- or higher dimensional problems. We have to stress that there are technical problems extending the result to general domains, especially when the boundary has a complicated geometry. In the proof for arbitrary domains we only need to replace the cosines by the corresponding eigenfunctions. We should then use the ergodic theorem to recover the convergence of the sums, but there is no guarantee that we can still do that, the main technical problem being that we cannot easily isolate the wave-number as an argument of the function. Moreover, even if we could, the reduction of the two-dimensional Birkhoff's ergodic theorem to the one-dimensional case is not always possible: we would need to prove a true two-dimensional ergodic theorem, but such results are available in the literature.

3. Proof of the main result. The main result is on one hand based on an application of the theorem by Edelman and Kostlan. On the other hand, for the asymptotic behavior we need the convergence of series over growing domains and the value of the limit. This is established by a two-dimensional weighted Birkhoff's ergodic theorem. There is a vast literature on ergodic theorems, and the theory is well developed, so we do not attempt to give an overview. We just refer to [21, 22] for ergodic theorems on abstract groups or subgroups of lattices.

In the following we first state the one-dimensional ergodic theorem, then give a direct proof for a weighted ergodic theorem on arbitrary domains based on the analogous result on squares (see, for example, [24]). We also give an elementary proof that the usual ergodic theorem implies (under some conditions on the weights) a weighted version. Let us remark that the results we need are not in the usual setting of weighted ergodic theorems, as, for example, in the theory of "good weights" (see, for example, $[3,4]$ ), because we allow the weights to grow and furthermore we change the normalizing constant in front of the sum.

3.1. Ergodic theorem. Given a $\sigma$-algebra, a transformation $T$ is said to be uniquely ergodic if it has a unique ergodic measure. The map $z \mapsto z+\alpha$ on the unit circle is uniquely ergodic if and only if $\alpha$ is irrational. In this case the unique ergodic measure is the Lebesgue measure.

Theorem 3.1 (Birkhoff's ergodic theorem; see [13]). Let $(X, \mu)$ be a probability space. If $T$ is $\mu$-invariant and ergodic and $g$ is integrable, then for a.e. $z \in X$

$$
\lim _{N \rightarrow \infty} \frac{1}{N} \sum_{k=1}^{N} g\left(T^{k}(z)\right)=\int_{X} g(\zeta) \mathrm{d} \mu(\zeta) .
$$


Moreover if $T$ is continuous and uniquely ergodic with measure $\mu$ and if $g$ is continuous, then (4) holds for all $z \in X$ (instead of a.e.).

Remark 3.2. Having the result to hold for all initial conditions is of paramount importance, as we require it to hold for some specific initial values. We will then look for uniquely ergodic transformations.

3.2. The weighted averaging condition. First we draft a necessary requirement for averaged weighted sums fulfilling an ergodic-type property on a rectangleshaped summation domain. Then we show that this can be used to obtain summation on more general domains. In our case this is a quarter-ring-shaped subset in $\mathbb{N}^{2}$.

After that we show that the sums we need to calculate all fulfill this requirement.

Weighted AVERAGING CONDition. Let $\left([0,1]^{d}, \lambda\right)$ be the probability space with the Lebesgue measure $\lambda$. We say that $\left(f,\left(a_{m}\right)\right)$ with $f:[0,1]^{d} \rightarrow \mathbb{R}$ continuous and extended by periodicity to $\mathbb{R}^{d}$ and $a_{m} \in \mathbb{R}$ fulfills the weighted averaging condition if for every $x^{0} \in[0,1]^{d}$, every $\alpha \in \mathbb{N}^{d}$, and

$$
Q_{L}=\bigotimes_{i=1}^{d}\left[1, \ldots, \alpha_{i} L\right] \cap \mathbb{N}^{d},
$$

the following assumption holds:

$$
\frac{1}{\sum_{m \in Q_{L}} a_{m}} \sum_{m \in Q_{L}} a_{m} \cdot f\left(m_{1} x_{1}^{0}, m_{2} x_{2}^{0}, \ldots, m_{d} x_{d}^{0}\right) \stackrel{L \rightarrow \infty}{\longrightarrow} \int_{[0,1]^{d}} f(x) \mathrm{d} x .
$$

For any open set $M \subset \mathbb{R}_{+}^{d}$ we define

$$
M_{L}=(L \cdot M) \cap \mathbb{N}^{d},
$$

the projection on the positive integers of its scaled version. We define

$$
\left|M_{L}\right|_{a}=\sum_{m \in M_{L}} a_{m}
$$

and denote by $\left|M_{L}\right|$ the cardinality of $M_{L}$.

GENERATION OF MEASURES. We require that the weights $a=\left(a_{m}\right)$ generate a measure $\lambda_{a}$ on $\mathbb{R}_{+}^{d}$ which is equivalent to the Lebesgue measure $\lambda$, i.e., there exists an $\alpha>0$ such that for each set with open interior $M \subset \mathbb{R}_{+}^{d}$,

$$
L^{-\alpha}\left|M_{L}\right|_{a} \stackrel{L \rightarrow \infty}{\longrightarrow} \lambda_{a}(M) .
$$

Let us remark that in general, we could get the result for a weaker assumption on the measure $\lambda_{a}$ than being equivalent to the Lebesgue measure. But as in all our examples this is the case, we assume this for simplicity of presentation.

The trivial example for the generation of measures is the constant weights $a_{k, l}=1$ that generate the Lebesgue measure with $\alpha=d$.

Example 3.3. An example that is used frequently later is $a_{k, l}=k^{2}$ for dimension $d=2$. Then by Riemann sum approximation

$$
L^{-4}\left|M_{L}\right|_{a}=L^{-4} \sum_{(k, l) \in L \cdot M} k^{2}=\sum_{(k, l) \in M \cap \frac{1}{L} \mathbb{N}^{2}} k^{2} L^{-2} \stackrel{L \rightarrow \infty}{\longrightarrow} \int_{M} \xi^{2} \mathrm{~d}(\xi, \eta) .
$$

Thus the measure $\lambda_{\left(k^{2}, 1\right)}$ generated by the weight has a Lebesgue-density $(\xi, \eta) \mapsto \xi^{2}$. As the density is up to the Lebesgue null set $\xi=0$ everywhere strictly positive, the measures $\lambda_{\left(k^{2}, 1\right)}$ and $\lambda$ are equivalent. 


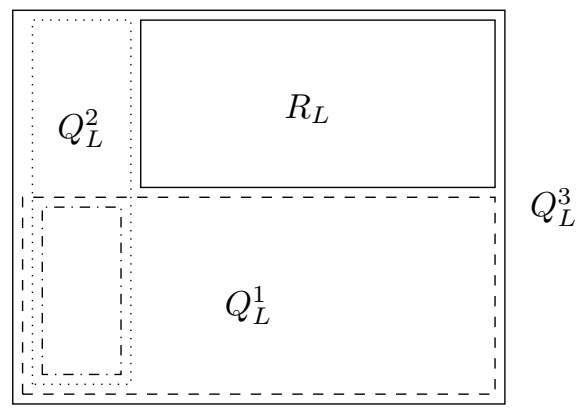

Fig. 5. Construction of $R_{L}$ from rectangles with one vertex in the origin: $R_{L}=Q_{L}^{3} \backslash\left(Q_{L}^{1} \cup Q_{L}^{2}\right)$.

Lemma 3.4. Let $\left(f,\left(a_{m}\right)\right)$ fulfill the weighted averaging condition with the weights a generating a measure. Then for any open measurable set $S \subset \mathbb{R}_{+}^{d}$

$$
\frac{1}{\left|S_{L}\right|_{a}} \sum_{m \in S_{L}} a_{m} \cdot f\left(m_{1} x_{1}^{0}, m_{2} x_{2}^{0}, \ldots, m_{d} x_{d}^{0}\right) \stackrel{L \rightarrow \infty}{\longrightarrow} \int_{[0,1]^{d}} f(x) \mathrm{d} x .
$$

Proof. We prove this in two steps. We call $S_{L}$ the summation domain. First we show that the weighted averaging condition also holds for summation domains which are rectangles not aligned at the origin (i.e., we shift the $Q_{L}$ out of the origin). Second, we cover $S$ by a disjoint union of such rectangles and conclude the proof. Consider the scaled rectangle $R_{L}=\otimes_{i=1}^{d}\left\{\beta_{i} L, \ldots, \gamma_{i} L\right\} \subset \mathbb{N}^{d}$. Then $R_{L}$ can be constructed by making unions and subtractions of origin-aligned rectangles of the form $Q_{L}$ as depicted in Figure 5. Thus

$$
\frac{1}{\left|R_{L}\right|_{a}} \sum_{m \in R_{L}} a_{m} \cdot f\left(m_{1} x_{1}^{0}, m_{2} x_{2}^{0}, \ldots, m_{d} x_{d}^{0}\right) \stackrel{L \rightarrow \infty}{\longrightarrow} \int_{[0,1]^{d}} f(x) \mathrm{d} x
$$

follows immediately.

For every approximation threshold $\delta>0$ there are finite sets of squares $\left\{P_{i}\right\}_{i}$ and $\left\{O_{i}\right\}_{i}$ fulfilling

$$
M_{\delta}=\bigcup_{i} P_{i} \subset S \subset \bigcup_{i} O_{i}=N_{\delta} .
$$

with $\lambda\left(N_{\delta} \backslash S\right) \leq \lambda\left(N_{\delta} \backslash M_{\delta}\right)<\delta$. Write $\left(M_{\delta, L}=M_{\delta} \cdot L\right) \cap \mathbb{N}^{d}, P_{i, L}=\left(P_{i} \cdot L\right) \cap$ $\mathbb{N}^{d}$, and $N_{\delta, L}=\left(N_{\delta} \cdot L\right) \cap \mathbb{N}^{d}$ analogously to $S$ and $S_{L}$. We write $f\left(m \odot x^{0}\right)=$ $f\left(m_{1} x_{1}^{0}, m_{2} x_{2}^{0}, \ldots, m_{d} x_{d}^{0}\right)$ for brevity. Then we can derive the error estimate

$$
\begin{aligned}
\operatorname{Err}=\left|\frac{1}{\left|N_{\delta, L}\right|_{a}} \sum_{m \in N_{\delta, L}} a_{m} f\left(m \odot x^{0}\right)-\frac{1}{\left|S_{L}\right|_{a}} \sum_{m \in S_{L}} a_{m} f\left(m \odot x^{0}\right)\right| \\
=\left.\frac{1}{\left|N_{\delta, L}\right|_{a} \cdot\left|S_{L}\right|_{a}}|| S_{L}\right|_{a} \sum_{m \in N_{\delta, L}} a_{m} f\left(m \odot x^{0}\right)-\left|N_{\delta, L}\right|_{a} \sum_{m \in S_{L}} a_{m} f\left(m \odot x^{0}\right) \mid \\
=\frac{1}{\left|N_{\delta, L}\right|_{a} \cdot\left|S_{L}\right|_{a}} \mid\left(\left|N_{\delta, L}\right|_{a}-\left|S_{L}\right|_{a}\right) \sum_{m \in S_{L}} a_{m} f\left(m \odot x^{0}\right) \\
+\left|S_{L}\right|_{a} \sum_{m \in N_{\delta, L} \backslash S_{L}} a_{m} f\left(m \odot x^{0}\right) \mid
\end{aligned}
$$




$$
\begin{aligned}
\leq & \frac{1}{\left|N_{\delta, L}\right|_{a} \cdot\left|S_{L}\right|_{a}}\left|\left(\left|N_{\delta, L}\right|_{a}-\left|S_{L}\right|_{a}\right)\|f\|_{\infty} \sum_{m \in S_{L}} a_{m}\right| \\
& +\left.\frac{1}{\left|N_{\delta, L}\right|_{a} \cdot\left|S_{L}\right|_{a}}|| S_{L}\right|_{a} \cdot\|f\|_{\infty} \sum_{m \in N_{\delta, L} \backslash S_{L}} a_{m} \mid \\
= & 2\|f\|_{\infty} \cdot \frac{\left.|| N_{\delta, L}\right|_{a}-\left|S_{L}\right|_{a} \mid}{\left|N_{\delta, L}\right|_{a}} \\
= & 2\|f\|_{\infty} \cdot \frac{\left|N_{\delta, L} \backslash S_{L}\right|_{a}}{\left|N_{\delta, L}\right|_{a}} \stackrel{L \rightarrow \infty}{\longrightarrow} 2\|f\|_{\infty} \cdot \frac{\lambda_{a}\left(N_{\delta} \backslash S\right)}{\lambda_{a}\left(N_{\delta}\right)} \stackrel{\delta \rightarrow 0}{\longrightarrow} 0,
\end{aligned}
$$

where in the last step we use that the weight $a$ generates a measure $\lambda_{a}$ equivalent to the Lebesgue measure. Thus $\lambda_{a}\left(N_{\delta}\right) \rightarrow \lambda_{a}(S)>0$ and $\lambda_{a}\left(N_{\delta} \backslash S\right) \rightarrow \lambda_{a}(\varnothing)=0$.

Hence the approximation error has for all $\delta>0$ a limit for $L \rightarrow \infty$ which is arbitrarily small for $\delta \rightarrow 0$, and it remains to check the limit on the cubical approximation $N_{\delta}$ instead of $S$, but this is straightforward:

$$
\begin{aligned}
& \frac{1}{\left|N_{\delta, L}\right|} \sum_{m \in M_{\delta, L}} f\left(m \odot x^{0}\right) \\
& =\frac{1}{\left|N_{\delta, L}\right|} \sum_{\substack{P_{i, L} \\
\cup_{i} P_{i, L}=N_{\delta, L}}}\left|P_{i, L}\right| \cdot \frac{1}{\left|P_{i, L}\right|} \sum_{m \in P_{i, L}} f\left(m \odot x^{0}\right) \stackrel{L \rightarrow \infty}{\longrightarrow} \int_{[0,1]^{d}} f(x) \mathrm{d} x,
\end{aligned}
$$

according to the first step of this proof.

3.3. Weighted averages. Here we present an elementary result showing that if the averages converge, then the weighted averages do, too.

Lemma 3.5. Given two sequences $\left\{b_{k}\right\}_{k}$ and $\left\{f_{k}\right\}_{k}$ such that $b_{k} \geq 0$ for all $k$, if

$$
F_{N}=\frac{1}{N} \sum_{k=1}^{N} f_{k} \stackrel{N \rightarrow \infty}{\longrightarrow} 0,
$$

and also $\frac{a_{N} \cdot N}{\sum_{k=1}^{N} a_{k}} \stackrel{N \rightarrow \infty}{\longrightarrow}$, for some $a \in \mathbb{R}$, with $a_{k}=\sum_{n=1}^{k} b_{n}$, and $\sum_{k=1}^{N} a_{k} \stackrel{N \rightarrow \infty}{\longrightarrow}$ $\infty$, then

Proof. Consider first

$$
\frac{\sum_{k=1}^{N} a_{k} \cdot f_{k}}{\sum_{k=1}^{N} a_{k}} \stackrel{N \rightarrow \infty}{\longrightarrow} 0
$$

$$
\sum_{n=1}^{N} n \cdot b_{n}=\sum_{n=1}^{N} b_{n} \cdot N-\sum_{n=1}^{N-1} 1 \cdot \sum_{k=1}^{n} b_{k}=N \cdot a_{N}-\sum_{n=1}^{N-1} a_{n}
$$

which means that

Now

$$
\frac{\sum_{n=1}^{N} n \cdot b_{n}}{\sum_{n=1}^{N} a_{n}} \stackrel{N \rightarrow \infty}{\longrightarrow} a-1 .
$$

$$
\begin{aligned}
\frac{\sum_{k=1}^{N} a_{k} f_{k}}{\sum_{k=1}^{N} a_{k}} & =\frac{\sum_{k=1}^{N} \sum_{n=1}^{k} b_{n} \cdot f_{k}}{\sum_{k=1}^{N} a_{k}} \\
& =\frac{\sum_{n=1}^{N} b_{n} \cdot \sum_{k=n}^{N} f_{k}}{\sum_{k=1}^{N} a_{k}}
\end{aligned}
$$




$$
\begin{aligned}
& =\frac{N}{\sum_{k=1}^{N} a_{k}} \sum_{n=1}^{N}\left[b_{n} \cdot F_{N}-b_{n} \cdot \frac{n}{N} \cdot F_{n}\right] \\
& =F_{N} \cdot \frac{N \cdot a_{N}}{\sum_{k=1}^{N} a_{k}}-\sum_{n=1}^{N} F_{n} \cdot \frac{n \cdot b_{n}}{\sum_{k=1}^{N} a_{k}} .
\end{aligned}
$$

Note that $\sum_{n=1}^{N} F_{n} \cdot \frac{n \cdot b_{n}}{\sum_{k=1}^{N} a_{k}}$ is bounded by a constant $C$ and there is $N_{1}$ such that $\left|F_{N} \cdot \frac{N \cdot a_{N}}{\sum_{k=1}^{N} a_{k}}\right|<\frac{\varepsilon}{3}$ for $n>N_{1}$. Also, there exists $N_{2}$ such that for $n>N_{2}$ we have

$$
\left|\sum_{n=1}^{N_{2}} F_{n} \cdot \frac{n \cdot b_{n}}{\sum_{k=1}^{N} a_{k}}\right| \leq C \cdot \frac{\sum_{k=1}^{N_{2}} a_{k}}{\sum_{k=1}^{N} a_{k}} \leq \frac{\varepsilon}{3} .
$$

Finally, we can choose $N_{3}$ such that for $N \geq N_{3}$

$$
\left|\sum_{n=1}^{N_{2}} F_{n} \cdot \frac{n \cdot b_{n}}{\sum_{k=1}^{N} a_{k}}\right|<\frac{\varepsilon}{3} .
$$

Then

$$
\begin{aligned}
\left|\frac{\sum_{k=1}^{N} a_{k} f_{k}}{\sum_{k=1}^{N} a_{k}}\right| & \leq\left|F_{N} \cdot \frac{N \cdot a_{N}}{\sum_{k=1}^{N} a_{k}}\right|+\left|\sum_{n=1}^{N_{2}} F_{n} \cdot \frac{n \cdot b_{n}}{\sum_{k=1}^{N} a_{k}}\right|+\left|\sum_{n=N_{2}+1}^{N} F_{n} \cdot \frac{n \cdot b_{n}}{\sum_{k=1}^{N} a_{k}}\right| \\
& <\varepsilon
\end{aligned}
$$

for $n>\max \left(N_{1}, N_{2}, N_{3}\right)$.

The following corollary follows immediately by setting $f=g-C$.

Corollary 3.6. Let the coefficients $a_{k}$ be as in Lemma 3.5. If

$$
\frac{1}{N} \sum_{k=1}^{N} g_{k} \stackrel{N \rightarrow \infty}{\longrightarrow} C,
$$

then

$$
\frac{\sum_{k=1}^{N} a_{k} \cdot g_{k}}{\sum_{k=1}^{N} a_{k}} \stackrel{N \rightarrow \infty}{\longrightarrow} C .
$$

3.4. Asymptotic behavior of the $\boldsymbol{S}_{\boldsymbol{i}}$. Now we turn to the main technical tool to prove the main result.

Lemma 3.7. For $x, \tau \in(0,1)$,

$$
\begin{gathered}
\lim _{\varepsilon \rightarrow 0} \frac{1}{\left|R_{\varepsilon}\right|} \cdot S_{1}=\frac{1}{4}, \\
\lim _{\varepsilon \rightarrow 0} \varepsilon^{2} \cdot \frac{S_{3}}{S_{1}}=\frac{1}{4}, \\
\lim _{\varepsilon \rightarrow 0} \varepsilon \cdot \frac{S_{2}}{S_{1}}=0 .
\end{gathered}
$$

Remark 3.8. In the following proof we will show that the weighted averaging condition holds for $x, \tau \notin \mathbb{Q}$ by using Birkhoff's ergodic theorem, Theorem 3.1. This leaves out the case when either $x$ or $\tau$ is in $\mathbb{Q}$. We have two ways of addressing this 
issue. We can compute explicitly the terms and obtain analytically the convergence required for the weighted average condition; these are straightforward but tedious computations, a sample of which is provided in Appendix A.

At the same time we can observe that it is enough to have the limits almost everywhere in $x, \tau$, as we are integrating $W_{\tau}(x)$ and the Lebesgue integral ignores sets of null measure.

Proof of Lemma 3.7. As discussed above, we will consider only the case $x, \tau \notin \mathbb{Q}$.

We first prove (5). We can use Birkhoff's ergodic theorem. Define $T_{x}(z)=z+x$ : this is a measure-preserving and uniquely ergodic transformation (since $x \notin \mathbb{Q}$ ). Then

$$
\frac{1}{N} \sum_{k=0}^{N-1} \cos ^{2}(\pi k x)=\frac{1}{N} \sum_{k=0}^{N-1} \cos ^{2}\left(\pi T_{x}^{k}(0)\right) \stackrel{N \rightarrow \infty}{\longrightarrow} \int_{0}^{1} \cos ^{2}(\pi x) \mathrm{d} x=\frac{1}{2} .
$$

All the coefficients $a_{m}$ are 1 in this case and the function is multiplicative. Then the result follows immediately from Lemma 3.4 and the fact that

$$
\int_{[0,1]^{2}} \cos ^{2}\left(\pi x_{1}\right) \cos ^{2}\left(\pi x_{2}\right) \mathrm{d}\left(x_{1}, x_{2}\right)=\frac{1}{4} .
$$

We prove now (6). For $x, \tau \notin \mathbb{Q}$ we know from (5) that the denominator $S_{1} \sim \frac{\left|R_{\varepsilon}\right|}{4}$ as $\varepsilon \rightarrow 0$ for $x \neq 0,1$. Hence the term in question has the same asymptotic behavior as

$$
\varepsilon^{2} \frac{S_{3}}{S_{1}} \sim 4 \pi^{2} \frac{\varepsilon^{2}}{\left|R_{\varepsilon}\right|} \cdot \sum_{k, l \in R_{\varepsilon}} k^{2} \sin ^{2}(k \pi x) \cos ^{2}(l \pi \tau) .
$$

We define $a=\left(a_{k, l}\right)_{k, l}$ with $a_{k, l}=k^{2}$, where we already saw in our example that this generates a measure with Lebesgue density such that

$$
\lim _{\varepsilon \rightarrow 0} \varepsilon^{2} \cdot \frac{\left|R_{\varepsilon}\right|_{a}}{\left|R_{\varepsilon}\right|}=\lim _{\varepsilon \rightarrow 0} \varepsilon^{2} \cdot \frac{1}{\left|R_{\varepsilon}\right|} \sum_{k, l \in R_{\varepsilon}} k^{2}=\frac{\lambda_{a}(R)}{\lambda(R)},
$$

where the rescaled domain is

$$
R=\left\{(\eta, \xi) \in \mathbb{R}^{2} \mid \alpha_{\ominus}<\sqrt{\xi^{2}+\eta^{2}}<\alpha_{\oplus}\right\}
$$

which gives

$$
R_{\varepsilon}=\varepsilon^{-1} R \cap \mathbb{N}^{2} .
$$

As $\mathrm{d} \lambda_{a}=\eta^{2} \mathrm{~d}(\eta, \xi)$ we obtain by elementary calculations using polar coordinates

$$
\begin{aligned}
\frac{\lambda_{a}(R)}{\lambda(R)} & =\frac{4}{\pi\left(\alpha_{\oplus}^{2}-\alpha_{\ominus}^{2}\right)} \int_{R} \eta^{2} \mathrm{~d}(\eta, \xi) \\
& =\frac{4}{\pi\left(\alpha_{\oplus}^{2}-\alpha_{\ominus}^{2}\right)} \int_{0}^{\pi / 2} \int_{\alpha_{\ominus}}^{\alpha_{\oplus}} r^{3} \cos (\varphi)^{2} \mathrm{~d} r \mathrm{~d} \varphi=\frac{1}{\alpha_{\oplus}^{2}-\alpha_{\ominus}^{2}} \int_{\alpha_{\ominus}}^{\alpha_{\oplus}} r^{3} \mathrm{~d} r \\
& =\frac{1}{4} \frac{\alpha_{\oplus}^{4}-\alpha_{\ominus}^{4}}{\alpha_{\oplus}^{2}-\alpha_{\ominus}^{2}}=\frac{1}{4}\left(\alpha_{\oplus}^{2}+\alpha_{\ominus}^{2}\right)=\frac{1}{4 \pi^{2}} .
\end{aligned}
$$

Combining (8) and (9), it remains to show that we can apply the averaged Birkhoff's ergodic theorem to the sum. We already saw that it is sufficient to check this on large 
rectangles containing the origin. We define $Q_{L}$ as in the weighted averaging condition for $\alpha \in(0, \infty)^{2}$ so that

$$
Q_{L}=\left[1, \alpha_{1} L\right] \times\left[1, \alpha_{2} L\right] \cap \mathbb{N}^{2}=I_{L}^{(1)} \times I_{L}^{(2)} \quad \text { with } \quad I_{L}^{(j)}=\left[1, \alpha_{j} L\right] \cap \mathbb{N} .
$$

Because of the rectangular shape we can split the sum

$$
\begin{aligned}
& \frac{1}{\left|Q_{L}\right|_{a}} \cdot \sum_{(k, l) \in Q_{L}} k^{2} \sin ^{2}(k \pi x) \cos ^{2}(l \pi \tau) \\
& =\frac{1}{\sum_{k \in I_{L}^{(1)}} k^{2}} \cdot \sum_{k \in I_{L}^{(1)}} k^{2} \sin ^{2}(k \pi x) \cdot \frac{1}{\sum_{l \in I_{L}^{(2)}}} \sum_{l \in I_{L}^{(2)}} \cos ^{2}(l \pi \tau) \\
& \quad \sim \frac{1}{\left|I_{L}^{(1)}\right|} \cdot \sum_{k \in I_{L}^{(1)}} \sin ^{2}(k \pi x) \cdot \frac{1}{\left|I_{L}^{(2)}\right|} \sum_{l \in I_{L}^{(2)}} \cos ^{2}(l \pi \tau) \\
& \quad \stackrel{L \rightarrow \infty}{\longrightarrow} \frac{1}{4}
\end{aligned}
$$

by the standard one-dimensional ergodic theorem, where we used Corollary 3.6 to remove the weights. We can now use Lemma 3.4 to extend this to the ring and obtain

$$
\varepsilon^{2} \cdot \frac{S_{3}}{S_{1}} \sim 4 \pi^{2} \varepsilon^{2} \cdot \frac{\left|R_{\varepsilon}\right|_{a}}{\left|R_{\varepsilon}\right|} \cdot \frac{1}{\left|R_{\varepsilon}\right|_{a}} \cdot \sum_{k, l \in R_{\varepsilon}} k^{2} \sin ^{2}(k \pi x) \cos ^{2}(l \pi \tau) \stackrel{\varepsilon \rightarrow 0}{\longrightarrow} \frac{1}{4},
$$

where we evaluated the value of the limit in (9).

Finally, we prove (7). As was the case in (6), we can calculate the sum of the coefficients, factor them out, and see that the orders of magnitude of $\varepsilon$ cancel out. Then everything is reduced to the integral

$$
\int_{[0,1]^{2}} \cos \left(\pi x_{1}\right) \sin \left(\pi x_{1}\right) \cos ^{2}\left(\pi x_{2}\right) \mathrm{d}\left(x_{1}, x_{2}\right)=0 .
$$

3.5. Proof of main result. We now have all the ingredients to complete the proof of Theorem 2.3.

Proof of Theorem 2.3. From Lemma 3.7 we know that

$$
W_{\tau}(x)=\frac{S_{3}}{S_{1}}-\left(\frac{S_{2}}{S_{1}}\right)^{2} \sim \frac{1}{4 \varepsilon^{2}} \quad \text { as } \varepsilon \rightarrow 0 .
$$

By Theorem 2.2, the number of expected zeros on the horizontal line $L_{\tau}$ of length 1 is, for a given $\varepsilon$,

$$
N=\frac{1}{\pi} \int_{0}^{1} \sqrt{\frac{1}{4 \varepsilon^{2}}} \mathrm{~d} x=\frac{1}{2 \pi \cdot \varepsilon} .
$$

This is the same as saying that the average pattern size is $\frac{1}{N}=2 \pi \varepsilon$.

4. General Fourier domains. We can consider domains in Fourier space that are different from the quarter-ring but still scale with $1 / \varepsilon$. For all these domains we obtain for the horizontal line that the average pattern size is again $C \varepsilon^{-1}$, but now for an explicit constant $C$ that depends only on the size of the Fourier domain measured with $\lambda_{a}$. 
Also the constant might be different along different directions (which are not horizontal) in case we lose the symmetry in $k$ and $l$ in the Fourier domain, but we will discuss that later in section 6.

On the other hand, for horizontal lines we can give an explicit constant, in terms of the measure $\lambda_{a}$, and for vertical lines we can argue by symmetry. Let us discuss a few explicit examples.

Consider the following mode domains, where we define as before for some $\gamma \in$ $(0,1)$

$$
\begin{aligned}
k_{1}= & \frac{\alpha_{\ominus}}{\varepsilon}=\sqrt{\frac{1-\sqrt{1-\gamma}}{2 \pi^{2} \varepsilon^{2}}} \text { and } k_{2}=\frac{\alpha_{\oplus}}{\varepsilon}=\sqrt{\frac{1+\sqrt{1-\gamma}}{2 \pi^{2} \varepsilon^{2}}} \\
R_{\varepsilon}^{\gamma} & =\left\{(k, l) \in \mathbb{N}^{2} \mid k_{1}<\sqrt{k^{2}+l^{2}}<k_{2}\right\}, \\
\mathcal{Q}_{\varepsilon}^{1} & =\left\{(k, l) \in \mathbb{N}^{2} \mid 0<k, l<k_{2}\right\}, \\
\mathcal{Q}_{\varepsilon}^{2} & =\left\{(k, l) \in \mathbb{N}^{2} \mid k_{1}<k, l<k_{2}\right\}, \\
\mathcal{Q}_{\varepsilon}^{3} & =\left\{(k, l) \in \mathbb{N}^{2} \mid k_{1}<k<k_{2}, 2 k_{1}<l<k_{1}+k_{2}\right\} .
\end{aligned}
$$

Note that $R_{\varepsilon}^{\gamma}$ is the same quarter-ring-shaped domain as before and the others are certain rectangles, all of them represented in Figure 6. For our main result, we will also use the scaled $\varepsilon$-independent versions

$$
\begin{gathered}
R^{\gamma}=\left\{(\eta, \xi) \in \mathbb{R}^{2} \mid \alpha_{\ominus}<\sqrt{\xi^{2}+\eta^{2}}<\alpha_{\oplus}\right\} \\
\mathcal{Q}^{1}=\left(0, \alpha_{\oplus}\right)^{2}, \quad \mathcal{Q}^{2}=\left(\alpha_{\ominus}, \alpha_{\oplus}\right)^{2}, \quad \mathcal{Q}^{3}=\left(\alpha_{\ominus}, \alpha_{\oplus}\right) \times\left(2 \alpha_{\ominus}, \alpha_{\ominus}+\alpha_{\oplus}\right),
\end{gathered}
$$
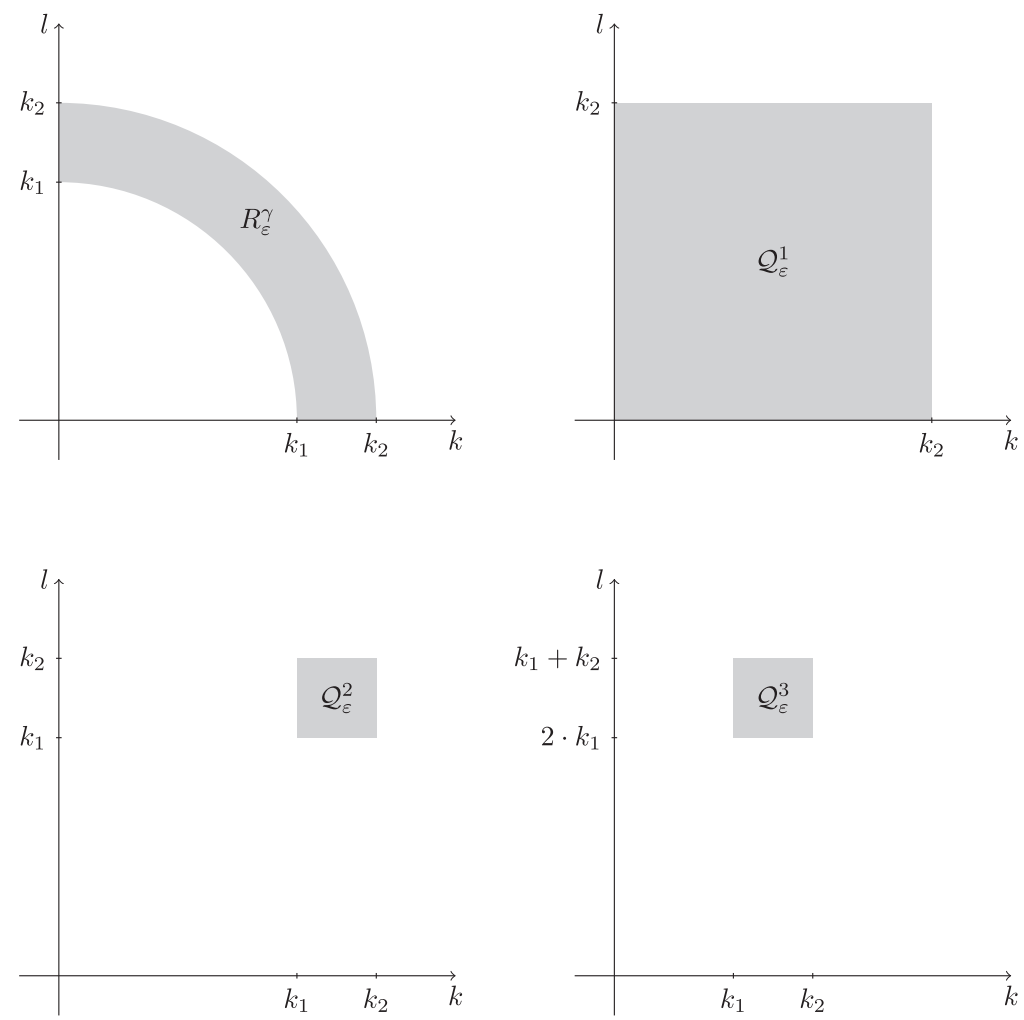

FIG. 6. The ring $R^{\gamma}$ and the alternative mode domains $\mathcal{Q}^{i}, i=1,2,3$. 
so that

$$
R_{\varepsilon}^{\gamma}=\varepsilon^{-1} R^{\gamma} \cap \mathbb{N}^{2} \quad \text { and } \quad \mathcal{Q}_{\varepsilon}^{i}=\varepsilon^{-1} \mathcal{Q}^{i} \cap \mathbb{N}^{2} \text { for } i=1,2,3 .
$$

We provide numerical simulations on what the patterns look like on such domains in Figure 7. They look quite different, although as we will see in the following Lemma 4.1, the main result about the asymptotic density of zeros is in all four cases $C \varepsilon$, only the constant $C$ changes from case to case.

Lemma 4.1. Let $D_{\varepsilon}=\varepsilon^{-1} D \cap \mathbb{N}^{2}$ be a scaled domain in Fourier space (for example $R_{\varepsilon}^{\gamma}$ or $\mathcal{Q}_{\varepsilon}^{i}$ for $i=1,2,3$ ). Then the asymptotic (for $\varepsilon \rightarrow 0$ ) density of zeros $\delta$ is on horizontal lines through the pattern

$$
\delta(x) \sim \frac{1}{2 \pi \varepsilon} \cdot \sqrt{\frac{\lambda_{\left(k^{2}, 1\right)}(D)}{\lambda(D)}},
$$

while on vertical lines it is

$$
\delta(x) \sim \frac{1}{2 \pi \varepsilon} \cdot \sqrt{\frac{\lambda_{\left(1, l^{2}\right)}(D)}{\lambda(D)}} .
$$

Proof. This is a proof analogous to the one of Lemma 3.7. We obtain the following asymptotic equivalences:

$$
\delta(x)^{2} \sim \frac{S_{3}}{S_{1}} \sim \frac{1}{4} \cdot \frac{\left|D_{\varepsilon}\right|_{\left(k^{2}, 1\right)}}{\left|D_{\varepsilon}\right|} \sim \frac{1}{4 \varepsilon^{2}} \cdot \frac{\lambda_{\left(k^{2}, 1\right)}(D)}{\lambda(D)} .
$$

The statement on vertical lines simply follows by symmetry.

The correction factors $\lambda_{a}(D) / \lambda(D)$ for the weights $a_{k, l}=k^{2}$ and the pattern sizes in the following domains are

\begin{tabular}{r|c|c|c}
\hline Domain & Correction coeff. & Avg. number of zeros & Avg. pattern size \\
\hline$R_{\varepsilon}^{\gamma}$ & 1 & $\frac{1}{2 \pi \varepsilon}$ & $2 \pi \varepsilon$ \\
$\mathcal{Q}_{\varepsilon}^{1}$ & $2 / 3(1+\sqrt{1-\gamma})$ & $\frac{\sqrt{2 / 3(1+\sqrt{1-\gamma})}}{2 \pi \varepsilon}$ & $\frac{2 \pi \varepsilon}{\sqrt{2 / 3(1+\sqrt{1-\gamma})}}$ \\
$\mathcal{Q}_{\varepsilon}^{2}$ & $2 / 3(2+\sqrt{\gamma})$ & $\frac{\sqrt{2 / 3(2+\sqrt{\gamma})}}{2 \pi \varepsilon}$ & $\frac{2 \pi \varepsilon}{\sqrt{2 / 3(2+\sqrt{\gamma})}}$ \\
\hline
\end{tabular}

These are, due to symmetry, the same results for horizontal or vertical lines, while for the nonsymmetric $\mathcal{Q}^{3}$ we have

\begin{tabular}{r|c|c|c}
\hline $\mathcal{Q}_{\varepsilon}^{3}$ & Correction coeff. & Avg. number of zeros & Avg. pattern size \\
\hline \multirow{2}{*}{ horizontal } & $2 / 3(2+\sqrt{\gamma})$ & $\frac{\sqrt{2 / 3(2+\sqrt{\gamma})}}{2 \pi \varepsilon}$ & $\frac{2 \pi \varepsilon}{\sqrt{2 / 3(2+\sqrt{\gamma})}}$ \\
& $2 / 3(8-6 \sqrt{1-\gamma}+4 \sqrt{\gamma})$ & $\frac{\sqrt{2 / 3(8-6 \sqrt{1-\gamma}+4 \sqrt{\gamma})}}{2 \pi \varepsilon}$ & $\frac{2 \pi \varepsilon}{\sqrt{2 / 3(8-6 \sqrt{1-\gamma}+4 \sqrt{\gamma})}}$ \\
\hline
\end{tabular}

In Figure 7 the simulations are run with $\gamma=0.7$ and $\varepsilon=10^{-2}$. In those cases we have the following rounded-off asymptotic values for the number of zeros on a line: 

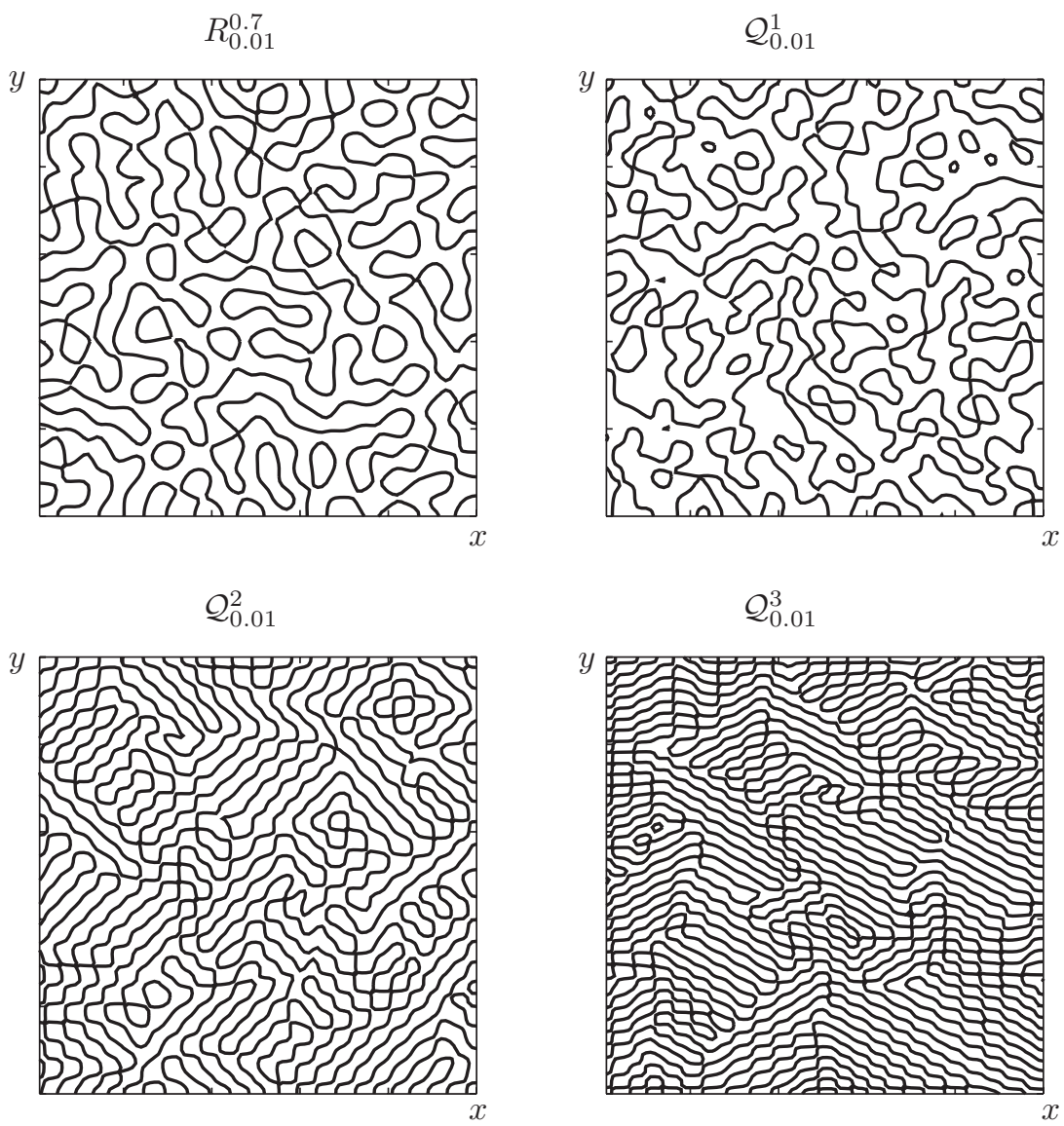

FIG. 7. Typical example of patterns generated by the Fourier domains presented in Figure 6. We cannot really say why these quite different pattern are observed, as our results just give $\mathcal{O}\left(\varepsilon^{-1}\right)$ for the asymptotic number of zeros along a straight line. It seems that the pattern gets more and more structured the smaller the domain is, and moreover the pattern size seems to decrease if the Fourier modes are on average further away from $(0,0)$.

\begin{tabular}{l|l|l|l}
\hline Domain & Correction coeff. & Avg. number of zeros & Avg. pattern size \\
\hline$R_{0.01}^{0.7}$ & 1 & $15.915(\times 1)$ & 0.062832 \\
$\mathcal{Q}_{0.01}^{1}$ & 1.032 & $16.167(\times 1.016)$ & 0.061856 \\
$\mathcal{Q}_{0.01}^{2}$ & 1.891 & $21.887(\times 1.375)$ & 0.045690 \\
$\mathcal{Q}_{0.01}^{3}$ (hor.) & 1.891 & $21.887(\times 1.375)$ & 0.045690 \\
$\mathcal{Q}_{0.01}^{3}$ (ver.) & 5.374 & $36.894(\times 2.318)$ & 0.027105. \\
\hline
\end{tabular}

The size of the correction coefficient is not that straightforward to explain. While $\mathcal{Q}_{1}$ is by far the largest domain, its correction is very close to 1 . At the same time for the smaller domains $\mathcal{Q}_{2}$ and $\mathcal{Q}_{3}$ the corrections are significantly larger than 1 . This can be explained by looking closer at the correction coefficient $\lambda_{\left(k^{2}, 1\right)}(D) / \lambda(D)$. For a fixed size $\lambda(D)$ of the domain, thanks to the weight $k^{2}$ we can increase the correction coefficient by moving the domain away from 0 . Moreover, if we move $D$ closer to the $k_{1}$-axis and further away from the $k_{2}$-axis, then also the correction grows. This is nicely visualized by the comparison of horizontal and vertical lines for $\mathcal{Q}_{3}$. The 
intuitive reasoning here is simple, as Fourier modes close to the $k_{1}$-axis have much faster oscillations in the $x$-direction than in the $y$-direction.

If we sample random horizontal lines in the numerical simulations represented in Figure 7, we get the following number of zeros and average pattern sizes, which are in good agreement with the predicted asymptotic results (the values are obtained by averaging over 500 simulations for each domain):

\begin{tabular}{l|cc|cc}
\hline & \multicolumn{2}{|c|}{ Avg. number of zeros } & \multicolumn{2}{c}{ Avg. pattern size } \\
Domain & Predicted & Sampled & Predicted & Sampled \\
\hline$R_{0.01}^{0.7}$ & 15.915 & 16.066 & 0.062832 & 0.060388 \\
$\mathcal{Q}_{0.01}^{1}$ & 16.167 & 16.502 & 0.061856 & 0.058540 \\
$\mathcal{Q}_{0.01}^{2}$ & 21.887 & 22.204 & 0.045690 & 0.044655 \\
$\mathcal{Q}_{0.01}^{3}$ (ver.) & 36.894 & 38.286 & 0.027105 & 0.026088. \\
\hline
\end{tabular}

It is surprising that the numerical result is in all four cases slightly biased in one direction, compared to the asymptotic result, but we have no explanation for this.

5. Numerical simulations. In Figure 8 we plot the (rescaled) density of zeros $\varepsilon \delta(x)=\frac{\varepsilon}{\pi} \sqrt{W_{\tau}(x)}$ for various values of $\varepsilon$ on the domain $R^{0.8}$. We scale by $\varepsilon$ so that the convergence to $1 / 2 \pi$ is easily seen. We can observe that the density in fact converges pointwise (apart from $x=0,1$ ). It even seems that the convergence is
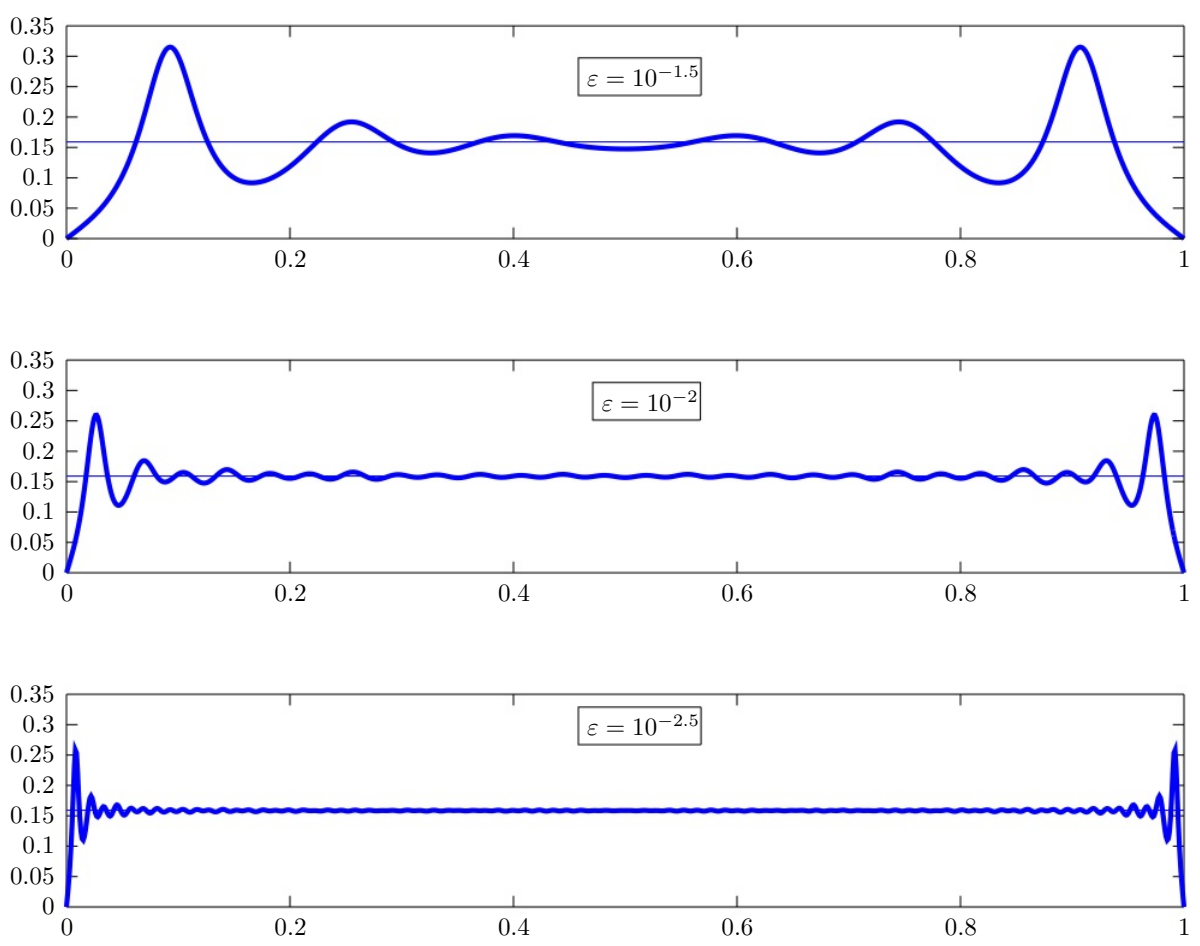

FIG. 8. Three examples of the (rescaled) density of zeros $\varepsilon \delta(x)$ for $\tau=0.5$ and $\gamma=0.8$ on the ring-shaped domain. These pictures are a graphical representation of the pointwise limit $\varepsilon \delta(x) \rightarrow \frac{1}{2 \pi}$ proven in Theorem 2.3. Inside the domain the convergence seems to be uniform and very fast. Only at the boundary the convergence is slow. 


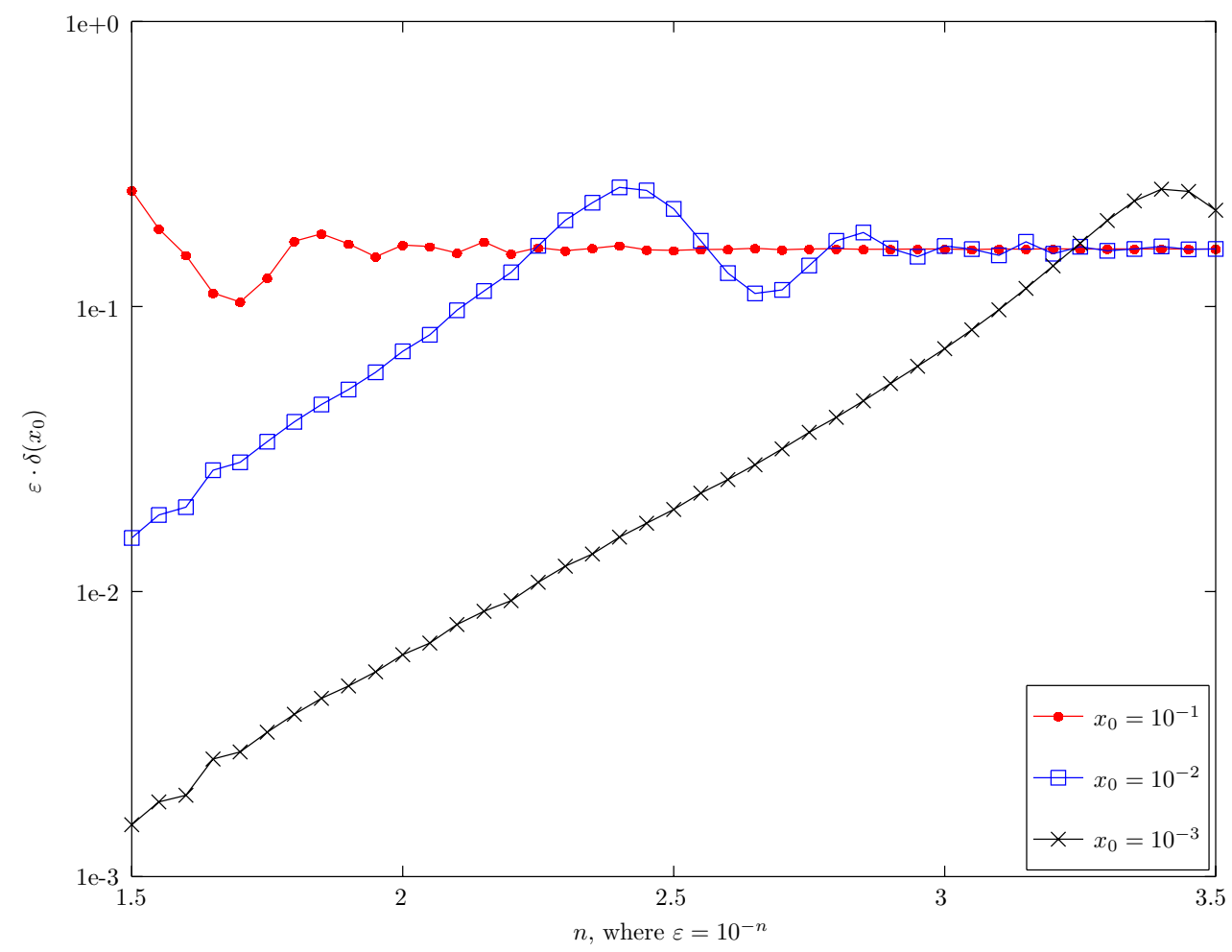

FIG. 9. Values for $\varepsilon \delta\left(x_{0}\right)$ for various values of $x_{0}$. The parameter $\varepsilon$ is in a logarithmic scale on the abscissa and the scaled value $\varepsilon \delta(x)$ of the density of zeros is on the ordinate.

uniform away from an arbitrarily small boundary layer and that the convergence is very fast. We do not have a heuristic explanation for this, as the speed of convergence comes from the ergodic theorem.

In order to see the speed of convergence, we fix a point $x_{0} \in(0,1)$ and track the value of $\varepsilon \delta\left(x_{0}\right)$ for $\varepsilon \rightarrow 0$. This is done in Figure 9.

Once again we can read off the convergence to $1 / 2 \pi$ but we can also quantify the convergence's disturbance by the "traveling wave" which goes to the borders of the interval: even if we choose a point which is very close to 0 (the critical points), for example, $x_{0}=10^{-2}$, we see that the magnitude of the density grows very quickly at first until it hits the limit value and then exhibits a damped oscillation around that asymptotic growth rate. When compared to Figure 8, we can imagine those traveling waves approaching and going through such a value $x_{0}$ until after some threshold $\varepsilon$, the oscillation is negligible.

5.1. Numerical comparison with the Cahn-Hilliard-Cook equation. As mentioned in the introduction, the average number of zeros along a line (horizontal, with correction term for sloped lines) is expected to be $1 / 2 \pi \varepsilon$ not only in our model but also for the actual Cahn-Hilliard-Cook equation. We will now provide numerical evidence in that sense.

To do so we use a standard semi-implicit spectral solver for the Cahn-HilliardCook equation to compute a solution of such equation seen as a time evolution up to some time $T$ which is still in the linear regime, as discussed in subsection 1.2. This solution depends in particular on the choice of the parameter $\varepsilon$. 
Once we have a solution at time $T$, we take a horizontal cut through the domain, in the same way as seen in Figure 3, and we find the zeros along that line. We have then the number of zeros along the line and the distances between two consecutive ones.

We can take a Monte Carlo approach and do this several times for each chosen value of the parameter $\varepsilon$, and then compare the (averaged) results obtained with those predicted from Theorem 2.3 and the (averaged) ones coming from simulations of the model, with the domain $R_{\varepsilon}^{\gamma}$ that best captures the essence of Cahn-Hilliard, as argued in subsection 1.2. The results for the number of zeros are presented in Table 1, while those for the distances between two consecutive zeros are in Table 2. An additional visual comparison is provided in Figures 10 and 11, where we plot, for the simulations of the model and of the Cahn-Hilliard-Cook equation, the number of zeros, and the average distance, respectively

TABLE 1

Average number of zeros, with standard deviation, for different values of $\varepsilon$. For the model we are considering the domain $R_{\varepsilon}^{\gamma}$ with $\gamma=0.7$. For the model, we ran 500 simulations for $\varepsilon=0.01$ and $\varepsilon=0.005$ and 100 simulations for $\varepsilon=0.001$. For simulation of the Cahn-Hilliard-Cook equation, we used 500, 500 and 100 iterations, respectively.

\begin{tabular}{l|c|c|c}
\hline$\varepsilon$ & Predicted & Simulated model & Simulated Cahn-Hilliard-Cook \\
\hline 0.01 & 15.915 & $16.066 \pm 2.307$ & $15.134 \pm 2.489$ \\
0.005 & 31.831 & $31.878 \pm 3.354$ & $29.776 \pm 3.401$ \\
0.001 & 159.150 & $159.690 \pm 7.150$ & $151.240 \pm 6.830$ \\
\hline
\end{tabular}

TABLE 2

Pattern size for different values of $\varepsilon$. For the model we are considering the domain $R_{\varepsilon}^{\gamma}$ with $\gamma=0.7$. For both the model and Cahn-Hilliard-Cook, we ran 500 simulations for $\varepsilon=0.01$ and $\varepsilon=0.005$ and 100 simulations for $\varepsilon=0.001$.

\begin{tabular}{c|c|cc|lc}
\hline & Predicted & \multicolumn{2}{|c|}{ Simulated model } & \multicolumn{2}{c}{ Simulated Cahn-Hilliard-Cook } \\
$\varepsilon$ & Average & Average & std & Average & std \\
\hline 0.01 & 0.062832 & 0.060388 & 0.033575 & 0.063650 & 0.037470 \\
0.005 & 0.031416 & 0.030787 & 0.018617 & 0.032922 & 0.019638 \\
0.001 & 0.0062832 & 0.0062375 & 0.0038216 & 0.006574 & 0.004077 \\
\hline
\end{tabular}

The theory we present here concerns only the expected number of zeros of a function in one dimension (which we apply to higher-dimensional solutions of the Cahn-Hilliard-Cook equation by making cuts through the domain). But the problem of getting an estimate on the pattern size is reciprocal to that. We can read Figure 11 not only horizontally, comparing the model and the Cahn-Hilliard-Cook equation, but also vertically, confronting the average pattern size measured directly (top), and as the reciprocal to the number of zeros (bottom). While not exactly the same (necessarily, given how they are obtained), they closely resemble each other.

6. Sloped lines. As we have already mentioned, the result given in Theorem 2.3 is proven only for horizontal lines and by symmetry for vertical lines. We claim that the result is far more general. In order to do so, we consider any sloped line through the origin. For the ring $R^{\gamma}$ we will see that we obtain the same asymptotic behavior.

To fix the setting we assume $y=\mu x$ with $\mu \in(0,1]$. This last assumption is just for simplicity of presentation, as we can use the reflection at the diagonal to get the analogous result for the remaining half square. 

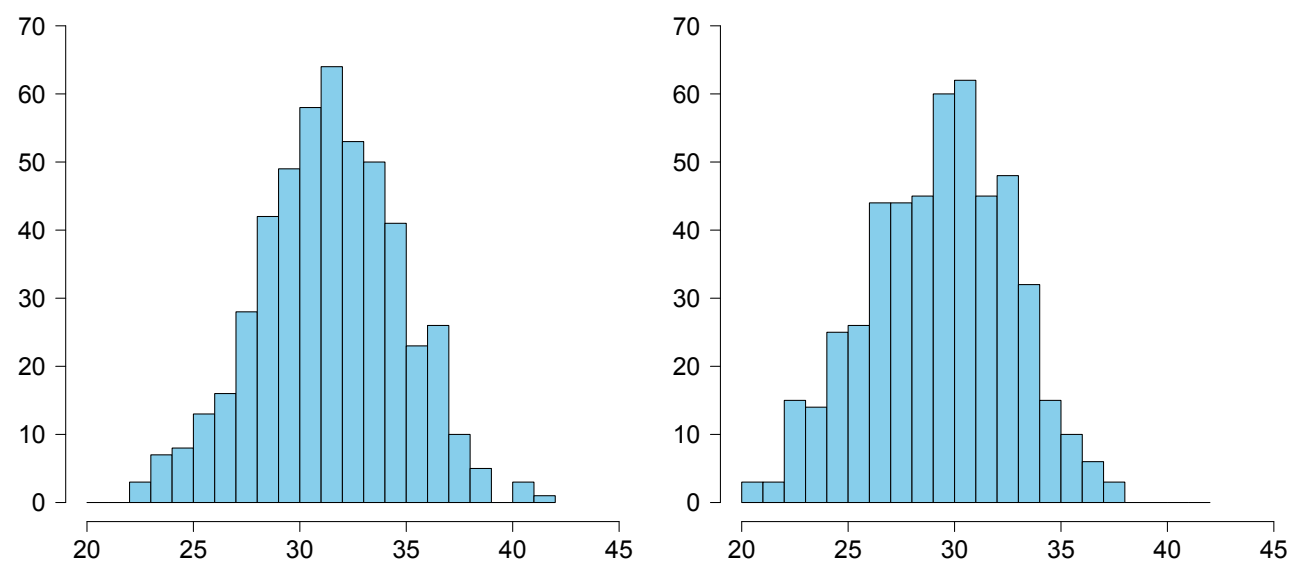

Fig. 10. Histograms of the number of zeros of the model (left) and of the Cahn-Hilliard-Cook equation (right). In both cases we ran 500 simulations setting the parameter $\varepsilon$ to 0.005 .
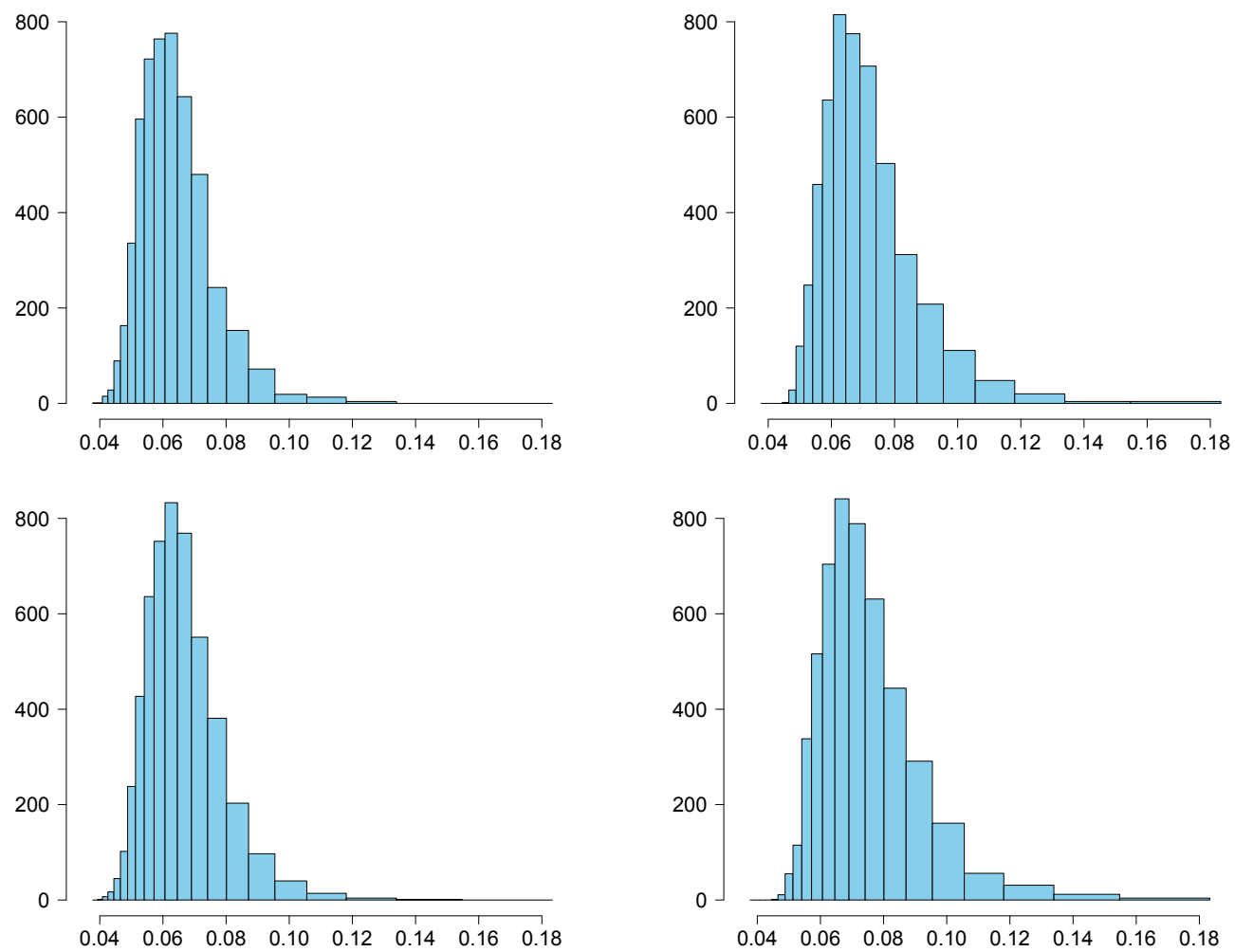

FIG. 11. Comparison of average "pattern size" obtained for both the model (left) and the CahnHilliard-Cook equation (right). In both cases we ran 5000 simulations, setting $\varepsilon=0.01$. The top figures show the average distance between subsequent zeros, directly measured, and the bottom figures depict the inverse of the number of zeros in each of the simulations. Note that bars are not equally spaced: they are centered in the inverses of the natural numbers 26 down to 6 , as the reciprocal of the number of zeros can only assume values of the form $1 / N$, where $N \in \mathbb{N}$, and in this way the plots are comparable with each other. 
Note that considering lines through the origin is not restrictive, we do this only for simplicity of presentation. The more general case $y=\mu x+\hat{\tau}$ behaves in the same way, using the trigonometric formulas for the cosine of a sum, which just adds to the number of the terms involved.

The important thing in that situation is to consider only the intersection $(x, \mu x+$ $\hat{\tau}) \cap[0,1]^{2}$, so we have to pay attention to the $x$ 's that are in our domain, and to the corresponding length of the segment we need for the renormalization.

For $y=\mu x$, we have, instead of $w_{\tau}(x)$, the following vector of functions:

$$
w_{\mu}(x)=\left(\cos (k \pi x) \cos (l \pi \mu x) /\left(\sum_{m, n} \cos ^{2}(m \pi x) \cos ^{2}(n \pi \mu x)\right)^{1 / 2}\right)_{k, l}
$$

and also

$$
\begin{aligned}
W_{\mu}(x) & =\frac{\sum_{k, l}\left[\begin{array}{l}
-S_{1}(k \pi \sin (k \pi x) \cos (l \pi \mu x)+l \pi \mu \sin (l \pi \mu x) \cos (k \pi x)) \\
+\cos (k \pi x) \cos (l \pi \mu x) \tilde{S}_{2}
\end{array}\right]^{2}}{\left(S_{1}\right)^{3}} \\
& =\frac{\sum_{k, l}(k \pi \sin (k \pi x) \cos (l \pi \mu x)+l \pi \mu \sin (l \pi \mu x) \cos (k \pi x))^{2}}{S_{1}}-\left(\frac{\tilde{S}_{2}}{S_{1}}\right)^{2} \\
& =\frac{\tilde{S}_{3}}{S_{1}}-\left(\frac{\tilde{S}_{2}}{S_{1}}\right)^{2},
\end{aligned}
$$

where $S_{1}$ is as in the horizontal case with $\tau:=\mu x$ and

$$
\begin{aligned}
& \tilde{S}_{2}=\sum_{k, l} \cos (k \pi x) \cos (l \pi \tau)(k \pi \sin (k \pi x) \cos (l \pi \tau)+l \pi \mu \sin (l \pi \tau) \cos (k \pi x)) \\
& \tilde{S}_{3}=\sum_{k, l}(k \pi \sin (k \pi x) \cos (l \pi \tau)+l \pi \mu \sin (l \pi \tau) \cos (k \pi x))^{2}
\end{aligned}
$$

which are the generalizations of the $S_{2}$ and $S_{3}$ encountered before. To prove that things work the same way in this case, we have to prove first the following:

$$
\lim _{\varepsilon \rightarrow 0} \varepsilon^{2}\left(\frac{\tilde{S}_{2}}{S_{1}}\right)^{2}=0
$$

This can be shown with arguments analogous to those for horizontal lines. The main new tool we need is that the averaging works for weights $(k, 1),(1, l)$, and $(k, l)$. Then we need to notice that any term with a sin-function averages in the limit to 0 in the Birkhoff's ergodic theorem. This implies (10).

Second, with the same argument, we see the following asymptotic equivalence:

$$
\varepsilon^{2} \frac{\tilde{S}_{3}}{S_{1}} \sim \varepsilon^{2} \frac{1}{S_{1}}\left(\sum_{k, l} k^{2} \pi^{2} \sin (k \pi x)^{2} \cos (l \pi \mu x)^{2}+\mu^{2} \sum_{k, l} l^{2} \pi^{2} \sin (l \pi \mu x)^{2} \cos (k \pi x)^{2}\right)
$$

This implies the following theorem.

TheOREM 6.1. For sloped lines $y=\mu x$ with $\mu \in[0.1]$ and general Fourierdomains $D$ we have that

$$
\delta(x)^{2} \sim \frac{1}{4 \varepsilon^{2}} \cdot \frac{1}{\lambda(D)}\left(\lambda_{\left(k^{2}, 1\right)}(D)+\mu^{2} \lambda_{\left(1, l^{2}\right)}(D)\right) .
$$


If the domain $D$ is symmetric with respect to the reflection along the diagonal, like, for example, the ring $R^{\gamma}$ or the squares $\mathcal{Q}_{1}$ and $\mathcal{Q}_{2}$, then the density of zeros $\delta(x)$ is compared to a horizontal line modified by a factor $\sqrt{1+\mu^{2}}$. But this just compensates for the length of the segment, which is not 1 as for the horizontal line, but $\sqrt{1+\mu^{2}}$. Now the two factors cancel and the average pattern size is for every sloped line the same as for the horizontal line. Thus we obtain that for any sloped line the average pattern size is the same and of order $\varepsilon$.

Let us discuss in more detail the case of $\mathcal{Q}_{2}$ and $\mathcal{Q}_{3}$, where the nodal lines seem to be diagonal. The interesting question is what happens if we consider a sloped line almost parallel to those pattern. First the average number of zeros is finite, which follows from the result by Edelman and Kostlan; thus the probability is zero that our line coincides on a whole interval with a nodal line and produces infinitely many zeros. Moreover even if the nodal line is almost diagonal it still bends and has oscillations on the scale of order $\varepsilon$. So even if our line is almost on the nodal line, the spacing of zeros will be of order $\varepsilon$. But even if the density of zeros is high at this place, the chances are high that our line in a place further away will fully miss nodal lines and will lie between them without hitting any zero there. But this does not contradict our main result for the average number of zeros along a line. It does not say that the zeros are evenly spaced along a line.

Appendix A. The rational case. In the proof of Lemma 3.7 we considered only irrational values of $x$ and $\tau$. This is sufficient because we are interested in the density on a set of Lebesgue measure 1 because we integrate it anyway. But as an additional consideration, for rational values, the periodicity of trigonometric functions yields a nonergodic averaging result of the same type as on the irrational numbers.

We restrict ourselves to a particularly easy case but the actual proof is a straightforward generalization.

Let $x=\frac{1}{n}$ and $N=l \cdot n$ for $l, n \in \mathbb{N}$ without loss of generalization and consider

$$
\begin{aligned}
\frac{1}{N} \sum_{k=1}^{N} \cos ^{2}(k \pi x) & =\frac{1}{2 N} \sum_{k=1}^{N} \cos \left(2 \pi k \frac{1}{n}\right) \\
& =\frac{1}{2}+\frac{1}{2} \cdot\left(\frac{1}{2} \frac{\sin \left(\frac{2 \pi N+\pi}{n}\right)-1}{\sin \left(\frac{\pi}{n}\right)}\right) \\
& =\frac{1}{2}-\frac{1}{4 N} \stackrel{N \rightarrow \infty}{\longrightarrow} \frac{1}{2} .
\end{aligned}
$$

This corresponds nicely to the ergodic result

$$
\lim _{N \rightarrow \infty} \frac{1}{N} \sum_{k=1}^{N} \cos ^{2}(k \pi x)=\frac{1}{2}
$$

for irrational $x$. In this way, we can, for example, prove

$$
\frac{1}{\left|R_{\varepsilon}\right|} S_{1}=\frac{1}{\left|R_{\varepsilon}\right|} \sum_{k, l \in R_{\varepsilon}} \cos ^{2}(k \pi x) \cos ^{2}(l \pi \tau) \stackrel{N \rightarrow \infty}{\longrightarrow} \frac{1}{4} .
$$

Acknowledgments. The authors want to thank Thomas Wanner for pointing out the result by Edelman and Kostlan. Moreover, the second and third authors would like to thank Evelyn Sander and Thomas Wanner for their hospitality. The authors would also like to thank the referees for the valuable suggestions to improve the paper. 


\section{REFERENCES}

[1] R. J. Adler and J. E. TaYlor, Random Fields and Geometry, Springer Monogr. Math, Springer, New York, 2007, https://doi.org/10.1007/978-0-387-48116-6.

[2] R. J. Adler and J. E. TAYlor, Topological Complexity of Smooth Random Functions. École d'Été de Probabilités de Saint-Flour XXXIX-2009, Lecture Notes in Math., 2019, Springer, Berlin, 2011, https://doi.org/10.1007/978-3-642-19580-8.

[3] J. Baxter And J. Olsen, Weighted and subsequential ergodic theorems, Canad. J. Math., 35, (1983), pp. 145-166, https://doi.org/10.4153/CJM-1983-010-7.

[4] A. Bellow And V. Losert, The weighted pointwise ergodic theorem and the individual ergodic theorem along subsequences, Trans. Amer. Math. Soc., 288 (1985), pp. 307-345, https://doi.org/10.1090/S0002-9947-1985-0773063-8.

[5] D. Blömker and S. Maier-PAape, Pattern formation below criticality forced by noise, Z. Angew. Math. Phys., 54 (2003), pp. 1-25, https://doi.org/10.1007/PL00012626.

[6] D. Blömker, S. Maier-PaApe, and T. Wanner, Spinodal decomposition for the CahnHilliard-Cook equation., Comm. Math. Phys., 223 (2001), pp. 553-582, https://doi.org/ 10.1007 /PL00005585.

[7] D. Blömker, S. Maier-PaApe, And T. Wanner, Phase separation in stochastic Cahn-Hilliard models, in Mathematical Methods and Models in Phase Transitions, A. Miranville, ed., Nova Science Publishers, New York, 2005, pp. 1-41.

[8] D. Blömker, S. Maier-PaApe, And T. Wanner, Second phase spinodal decomposition for the Cahn-Hilliard-Cook equation, Trans. Amer. Math. Soc., 360 (2008), pp. 449-489, https: //doi.org/10.1090/S0002-9947-07-04387-5.

[9] D. BlÖmker And M. Romito, Stochastic PDEs and lack of regularity: A surface growth equation with noise: existence, uniqueness, and blow-up, Jahresber. Dsch. Math. Ver., 117 (2015), pp. 233-286, https://doi.org/10.1365/s13291-015-0123-0.

[10] J. W. CAhn, Free energy of a nonuniform system, II. Thermodynamic basis, J. Chem. Phys., 30 (1959), pp. 1121-1124, https://doi.org/10.1063/1.1730145.

[11] J. W. Cahn And J. E. Hilliard, Free energy of a nonuniform system, I. Interfacial free energy, J. Chem. Phys., 28 (1958), pp. 258-267, https://doi.org/10.1063/1.1744102.

[12] H. Cook, Brownian Motion in Spinodal Decomposition, Acta Metallurgica, 18 (1970), pp. 297-306, https://doi.org/10.1016/0001-6160(70)90144-6.

[13] I. P. Cornfeld, S. V. Fomin, And Y. G. Sinai, Ergodic Theory, Grundlehren Math. Wiss., 245, Springer, Berlin, 1982.

[14] M. C. Cross and P. C. Hohenberg, Pattern formation outside of equilibrium, Rev. Modern Phys., 65 (1993), p. 851, https://doi.org/10.1103/RevModPhys.65.851.

[15] G. Da Prato and J. Zabczyk, Stochastic Equations in Infinite Dimensions, 2nd ed., Encyclopedia Math. Appl. 152, Cambridge University Press, Cambrdige, UK, 2014.

[16] S. Day, W. Kalies, K. Mischaikow, and T. Wanner, Probabilistic and numerical validation of homology computations for nodal domains, Electron. Res. Announc. Amer. Math. Soc., 13 (2007), pp. 60-73, https://doi.org/10.1090/S1079-6762-07-00175-8.

[17] S. Day, W. Kalies, and T. Wanner, Verified homology computations for nodal domains, Multiscale Model. Simul., 7 (2009), pp. 1695-1726, https://doi.org/10.1137/080735722.

[18] M. Dennis, Nodal densities of planar Gaussian random waves, Eur. Phys. J. Special Topics, 145 (2007), pp. 191-210, https://doi.org/10.1140/epjst/e2007-00156-1.

[19] A. Edelman and E. Kostlan, How many zeros of a random polynomial are real?, Bull. Amer. Math. Soc., 32 (1995), pp. 1-37, https://doi.org/10.1090/S0273-0979-1995-00571-9.

[20] M. Gameiro, K. Mischaikow, and T. Wanner, Evolution of pattern complexity in the CahnHilliard theory of phase separation, Acta Materialia, 53 (2005), pp. 693-704, https://doi. org/10.1016/j.actamat.2004.10.022.

[21] A. Gorodnik and A. Nevo, The Ergodic Theory of Lattice Subgroups, Princeton University Press, Princeton, NJ, 2009, https://arxiv.org/abs/0605596.

[22] A. Gorodnik And A. Nevo, Quantitative ergodic theorems and their number-theoretic applications, Bull. Amer. Math. Soc. (N.S.), 52 (2015), pp. 65-113, https://doi.org/10.1090/ S0273-0979-2014-01462-4.

[23] Y. Guo and H. J. Hwang, Pattern formation. II: The Turing instability, Proc. Amer. Math. Soc., 135 (2007), pp. 2855-2866, https://doi.org/10.1090/S0002-9939-07-08850-8.

[24] D. L. Hanson and G. Pledger, On the mean ergodic theorem for weighted averages, Z. Wahrscheinlichkeitstheorie Verwandte Gebiete, 13 (1969), pp. 141-149, https://doi.org/ 10.1007/BF00537020.

[25] R. Hoppe AND E. NASH, A combined spectral element/finite element approach to the numerical solution of a nonlinear evolution equation describing amorphous surface growth of thin films, J. Numer. Math., 100 (2002), pp. 127-136, https://doi.org/10.1515/JNMA.2002.127. 
[26] M. S. Longuet-Higgins, The statistical analysis of a random, moving surface, Philos. Trans. R. Soc. Lond. Ser. A Math. Phys. Eng. Sci., 249 (1957), pp. 321-387, https://doi.org/10. 1098/rsta.1957.0002.

[27] M. S. Longuet-Higgins, Statistical properties of an isotropic random surface, Philos. Trans. R. Soc. Lond. Ser. A Math. Phys. Eng. Sci., 250 (1957), pp. 157-174, https://doi.org/10. 1098/rsta.1957.0018.

[28] S. Maier-PaApe And T. Wanner, Spinodal decomposition for the Cahn-Hilliard equation in higher dimensions, Part I: Probability and wavelength estimate, Comm. Math. Phys., 195 (1998), pp. 435-464, https://doi.org/10.1007/s002200050397.

[29] S. Maier-PaApe And T. WANnER, Spinodal decomposition for the Cahn-Hilliard equation in higher dimensions: Nonlinear dynamics, Arch. Ration. Mech. Anal., 151 (2000), pp. 187219, https://doi.org/10.1007/s002050050196.

[30] J. Muñoz-García, R. Gago, L. Vázquez, J. A. Sánchez-García, and R. Cuerno, Observation and modeling of interrupted pattern coarsening: Surface nanostructuring by ion erosion, Phys. Rev. Lett., 104 (2010), 026101, https://doi.org/10.1103/PhysRevLett.104. 026101.

[31] J. D. Murray, Math. Biology II: Spatial Models and Biomedical Applications, Interdiscip. Appl. Math. 18, Springer, New York, 2003.

[32] M. Raible, S. Linz, And P. HänGGi, Amorphous thin film growth: Effects of density inhomogeneities, Phys. Rev. E, 64 (2001), 031506, https://doi.org/10.1103/PhysRevE.64.031506.

[33] S. O. RICE, Statistical properties of a sine wave plus random noise, Bell System Tech. J., 27 (1948), pp. 109-157, https://doi.org/10.1002/j.1538-7305.1948.tb01334.x.

[34] E. Sander And R. Tatum, Pattern formation in a mixed local and nonlocal reaction-diffusion system, Electron. J. Differential Equations, 2012 (2012), p. 30, http://ejde.math.txstate. edu/Volumes/2012/160/abstr.html.

[35] E. SANDER AND T. WANNER, Unexpectedly linear behavior for the Cahn-Hilliard equation, SIAM J. Appl. Math., 60 (2000), pp. 2182-2202, https://doi.org/10.1137/S0036139999352225.

[36] E. Sander And T. Wanner, Pattern formation in a nonlinear model for animal coats., J. Differential Equations, 191 (2003), pp. 143-174, https://doi.org/10.1016/S0022-0396(02) 00156-0.

[37] E. Siero, A. Doelman, M. B. Eppinga, J. D. M. Rademacher, M. Rietkerk, and K. Siteur, Striped pattern selection by advective reaction-diffusion systems: Resilience of banded vegetation on slopes., Chaos, 25 (2015), 036411, https://doi.org/10.1063/1.4914450.

[38] K. Siteur, E. Siero, M. Eppinga, J. D. M. Rademacher, A. Doelman, and M. Rietkerk, Beyond Turing: The response of patterned ecosystems to environmental change, Ecol. Complexity, 20 (2014), pp. 81-96, https://doi.org/10.1016/j.ecocom.2014.09.002.

[39] M. Sodin, Lectures on Random Nodal Portraits, 2012, http://www.math.tau.ac.il/ $\sim_{\text {sodin/ }}$ SPB-Lecture-Notes.pdf.

[40] T. WANNER, Maximum norms of random sums and transient pattern formation, Trans. Amer. Math. Soc., 356 (2003), pp. 2251-2279, https://doi.org/10.1090/S0002-9947-03-03480-9. 